\title{
Self-Guiding Polymeric Prodrug Micelles with Two Aggregation-Induced Emission Photosensitizers for Enhanced Chemo- Photodynamic Therapy
}

Xiaoqing Yi, ${ }^{\dagger, a, b}$ Jing-Jing Hu, ${ }^{\dagger, a} J u n$ Dai, ${ }^{c}$ Xiaoding Lou, ${ }^{*, a}$ Zujin Zhao, ${ }^{e}$ Fan Xia, ${ }^{*, a}$ and Ben Zhong Tang*,d,e

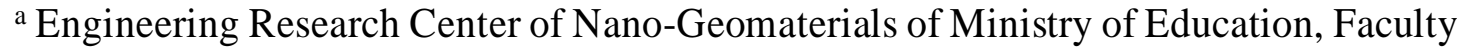
of Materials Science and Chemistry, China University of Geosciences, Wuhan 430074, China.

${ }^{\mathrm{b}}$ Key Laboratory of Prevention and Treatment of Cardiovascular and Cerebrovascular Diseases, Ministry of Education, Key Laboratory of Biomaterials and Biofabrication in Tissue Engineering of Jiangxi Province, Gannan Medical University, Ganzhou 341000, China.

c Department of Obstetrics and Gynecology, Tongji Hospital, Tongji Medical College, Huazhong University of Science and Technology, Wuhan 430074, China.

d Department of Chemistry, Hong Kong Branch of Chinese National Engineering Research Center for Tissue Restoration and Reconstruction, The Hong Kong University of Science and Technology, Clear Water Bay, Kowloon, Hong Kong, China 
e State Key Laboratory of Luminescent Materials and Devices, Guangdong Provincial Key Laboratory of Luminescence from Molecular Aggregates, South China University of Technology, Guangzhou 510640, China.

* Corresponding author. E-mail: louxiaoding@cug.edu.cn (X. Lou).

xiafan@cug.edu.cn (F.Xia).

tangbenz@ust.hk (B. Z. Tang).

${ }^{\dagger}$ These authors contributed equally to this work. 


\section{Table of Contents}

Table S1. Properties of PMPT and TB@PMPT micelles

Table S2. The slope of decomposition curves of ABDA induced by ROS generation from TB@PMP, PMPT and TB@PMPT micelles.

Figure S1. The main synthetic routes of PMPT.

Figure S2. ${ }^{1} \mathrm{H}$ NMR spectrum of 5-mthyl-5-propargyl-1,3-dioxan-2-one (MPMC).

Figure S3. ${ }^{1} \mathrm{H}$ NMR spectrum of PM.

Figure S4. ${ }^{1} \mathrm{H}$ NMR spectrum of PTX.

Figure S5. ${ }^{1} \mathrm{H}$ NMR spectrum of PTX-SS-N3.

Figure S6. ${ }^{1} \mathrm{H}$ NMR spectrum of PyTPE.

Figure S7. Mass spectrum of PyTPE.

Figure S8. ${ }^{1} \mathrm{H}$ NMR spectrum of PMPT.

Figure S9. Gel permeation chromatography trace of PMPT.

Figure S10. The TEM images PMPT and TB@PMPT micelles.

Figure S11. Hydrodynamic size distribution of PMPT and TB@PMPT micelles.

Figure S12. UV-vis spectrum of PMPT and TB@PMPT micelles.

Figure S13. Fluorescence spectra of PMPT and TB@PMP micelles.

Figure S14. UV-vis spectra of ABDA mixed with TB@PMP, PMPT, TB@PMPT and TB@PMP micelles upon light irradiation, respectively.

Figure S15. Detection of intracellular ROS production in HeLa cells after incubation with TB@PMP, PMPT and TB@PMPT micelles in the dark, respectively.

Figure S16. Corresponding quantitative analysis of the fluorescence intensity in Figure $1 \mathrm{~B}$.

Figure S17. CLSM images of HeLa cells after incubation with TB@PM and TB@PMPT micelles.

Figure S18. CLSM images of HeLa cells after incubation with TB@PMPT micelles with the first light irradiation $\left(\mathrm{L}_{1}\right)$ for different times.

Figure S19. Mass spectra of the released products from PTX-SS-N3 with the treatment of $10 \mathrm{mM}$ DTT.

Figure S20.The release mechanism of free PTX from PTX-SH under the condition of $10 \mathrm{mM}$ DTT.

Figure S21. The CMC value after PMPT micelles incubated with the condition of 0,2 , 10 and $50 \mathrm{mM}$ DTT, respectively.

Figure S22. Mass spectra of the released products from TB@PMPT micelles with the treatment of $10 \mathrm{mM}$ DTT. 
Figure S23. The size changes of (A) PMPT and (B) TB@PMPT micelles with TB loading of $9.57 \%$ in response to $10 \mathrm{mM}$ DTT at $\mathrm{pH} 7.4$

Figure S24. Mass spectra of the cell extract after incubation of TB@PMPT micelles with HeLa cells.

Figure S25.Fluorescence spectra and fluorescence intensity ratios of $I_{\mathrm{t}} / I_{0}$ and $I_{0} / I_{\mathrm{t}}$ of PMPT micelles (100 $\mathrm{mg} \mathrm{L}^{-1}$ ) was incubated with $10 \mathrm{mM}$ DTT for different times.

Figure S26. Fluorescence spectra and fluorescence intensity ratios of $I_{t} / I_{0}$ and $I_{0} / I_{\mathrm{t}}$ of PMPT micelles (200 $\mathrm{mg} \mathrm{L}^{-1}$ ) was incubated with $10 \mathrm{mM}$ DTT for different times.

Figure S27. Fluorescence spectra of TB@PMPT micelles with different concentrations and different TB loading content incubated with $10 \mathrm{mM}$ DTT for different times.

Figure S28. The fluorescence signal of PyTPE was observed by CLSM after PMPT micelles were incubated with HeLa cells for 2 hours.

Figure S29. The fluorescence signal of PyTPE and TB was observed by CLSM after TB@PMPT micelles were incubated with HeLa cells pretreated with GSH-OEt.

Figure S30. The fluorescence signal of PyTPE and TB was observed by CLSM after TB@PMPT micelles were incubated with HeLa cells pretreated with BSO.

Figure S31. CCK-8 assay of blank HeLa cells (LL, $\left.\mathrm{L}_{1} \mathrm{~L}_{2}\right)$ in HeLa cells after incubation for $48 \mathrm{~h}$.

Figure S32. CCK-8 assay of free PTX (-), PMPT (-) and TB@PMPT (-) in HeLa cells after incubation for $48 \mathrm{~h}$.

Figure S33. The inhibition ratios of TB@PM ( $\left.\mathrm{L}_{1} \mathrm{~L}_{2}\right)$, PMPT $\left(\mathrm{L}_{1} \mathrm{~L}_{2}\right)$, and TB@PMPT $\left(\mathrm{L}_{1} \mathrm{~L}_{2}\right)$ micelles treated cells upon light irradiation, respectively.

Figure S34. The inhibition ratios of TB@PM (LL), PMPT (LL), and TB@PMPT (LL) micelles treated cells, respectively.

Figure S35. Hemolysis assay after red blood cells incubation with different concentration of PMPT and TB@PMPT micelles.

Figure S36. Optical microscopic observation of the dispersion states of the erythrocytes. Figure S37. Body weight in the mice after the intravenous of different samples: PBS (-), TB@PMPT (-), TB@PMPT (LL), and TB@PMPT ( $\left.\mathrm{L}_{1} \mathrm{~L}_{2}\right)$.

Figure S38. H\&E staining images of the tumor and organs after injection of PBS (-), TB@PMPT (-), TB@PMPT (LL) and TB@PMPT ( $\left.\mathrm{L}_{1} \mathrm{~L}_{2}\right)$ micelles, respectively. 
Table S1. Properties of PMPT and TB@PMPT micelles

\begin{tabular}{llllll}
\hline \multirow{2}{*}{ Samples } & \multicolumn{3}{c}{ DLC (wt\%) } & \multirow{2}{*}{ Size $(\mathrm{nm})$} & \multirow{2}{*}{ PDI } \\
\cline { 2 - 4 } & PyTPE & PTX & TB & & \\
\hline PMPT & 7.35 & 18.5 & $/^{\mathrm{c}}$ & $116.8 \pm 2.5$ & $0.142 \pm 0.017$ \\
TB@PM & $/^{\mathrm{a}}$ & $/^{\mathrm{b}}$ & 7.25 & $60.2 \pm 1.5$ & $0.121 \pm 0.016$ \\
TB@PMP & $/^{\mathrm{a}}$ & 17.3 & 9.34 & $73.4 \pm 2.3$ & $0.143 \pm 0.015$ \\
TB@PMPT & 6.79 & 17.1 & 7.57 & $125.7 \pm 3.3$ & $0.112 \pm 0.025$ \\
TB@PMPT & 6.65 & 16.7 & 9.57 & $132.9 \pm 1.6$ & $0.120 \pm 0.047$ \\
TB@PMPT & 6.55 & 16.5 & 10.8 & $148.5 \pm 0.6$ & $0.087 \pm 0.024$ \\
\hline
\end{tabular}

${ }^{a}$ No PyTPE was conjugated on backbone of amphiphilic polycarbonate.

${ }^{\mathrm{b}}$ No PTX was encapsulated into PMPT micelles.

${ }^{\mathrm{c}}$ No TB was encapsulated into PMPT micelles. 
Table S2. The slope of decomposition curves of ABDA induced by ROS generation from TB@PMP, PMPT and TB@PMPT with or without 10 mM DTT were preincubated at $37^{\circ} \mathrm{C}$ for $1 \mathrm{~h}$.

\begin{tabular}{ccccccc}
\hline Samples & TB@PMP & PMPT & TB@PMPT & $\begin{array}{c}\text { TB@PMP } \\
\text { DTT, 1 h }\end{array}$ & $\begin{array}{c}\text { PMPT } \\
\text { DTT, 1 h }\end{array}$ & $\begin{array}{c}\text { TB@PMPT } \\
\text { DTT, 1 h }\end{array}$ \\
\hline Slope & 0.07347 & 0.08902 & 0.11750 & 0.08153 & 0.04521 & 0.08811 \\
Standard error & 0.00190 & 0.00198 & 0.00244 & 0.00236 & 0.00028 & 0.00237 \\
\hline
\end{tabular}




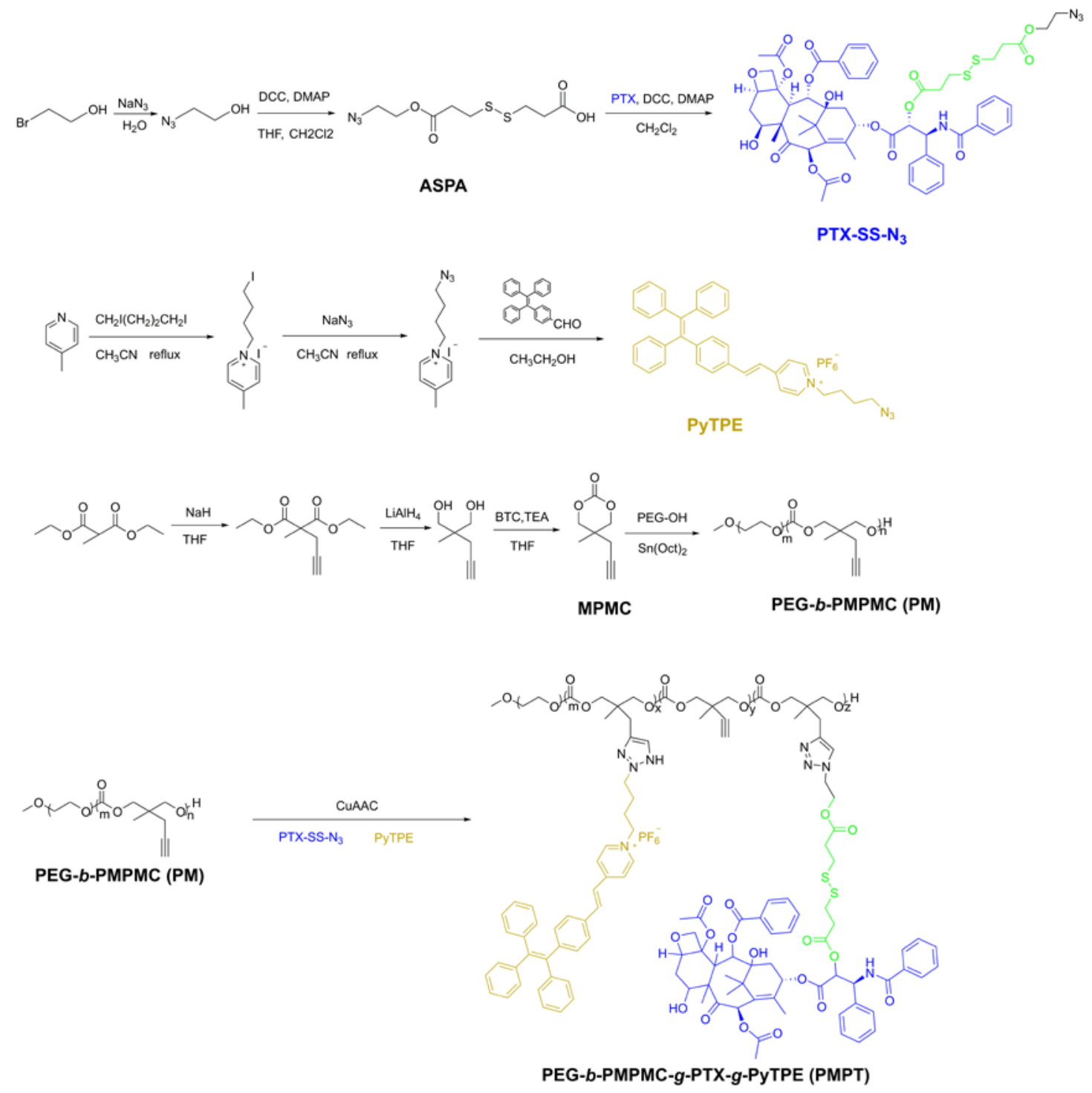

Figure S1. Synthesis of reduction-sensitive amphiphilic polymeric prodrug PMPT. 


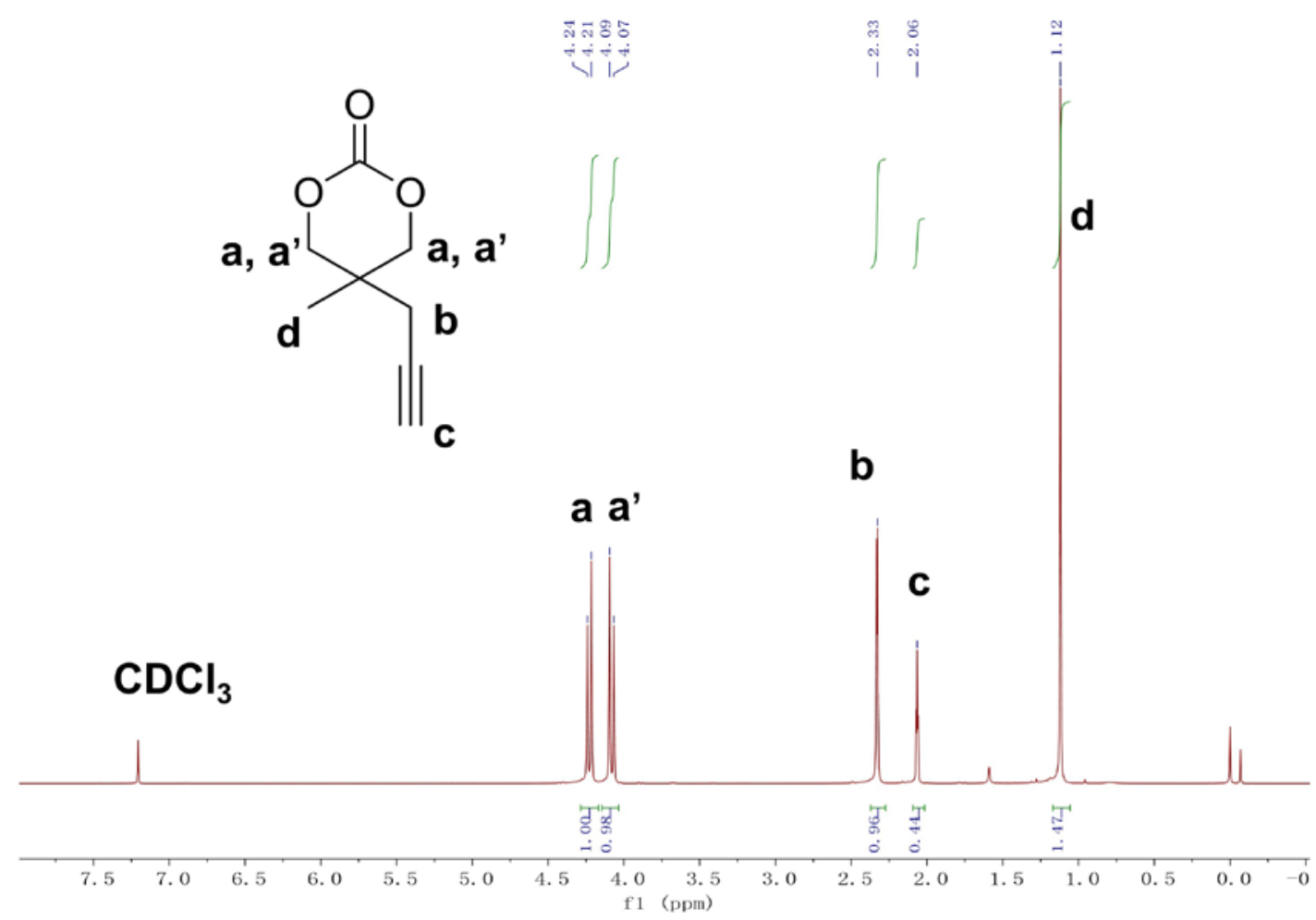

Figure S2. ${ }^{1} \mathrm{H}$ NMR spectrum (400 MHz, $\left.\mathrm{CDCl}_{3}\right)$ of 5-mthyl-5-propargyl-1,3-dioxan2-one (MPMC). 


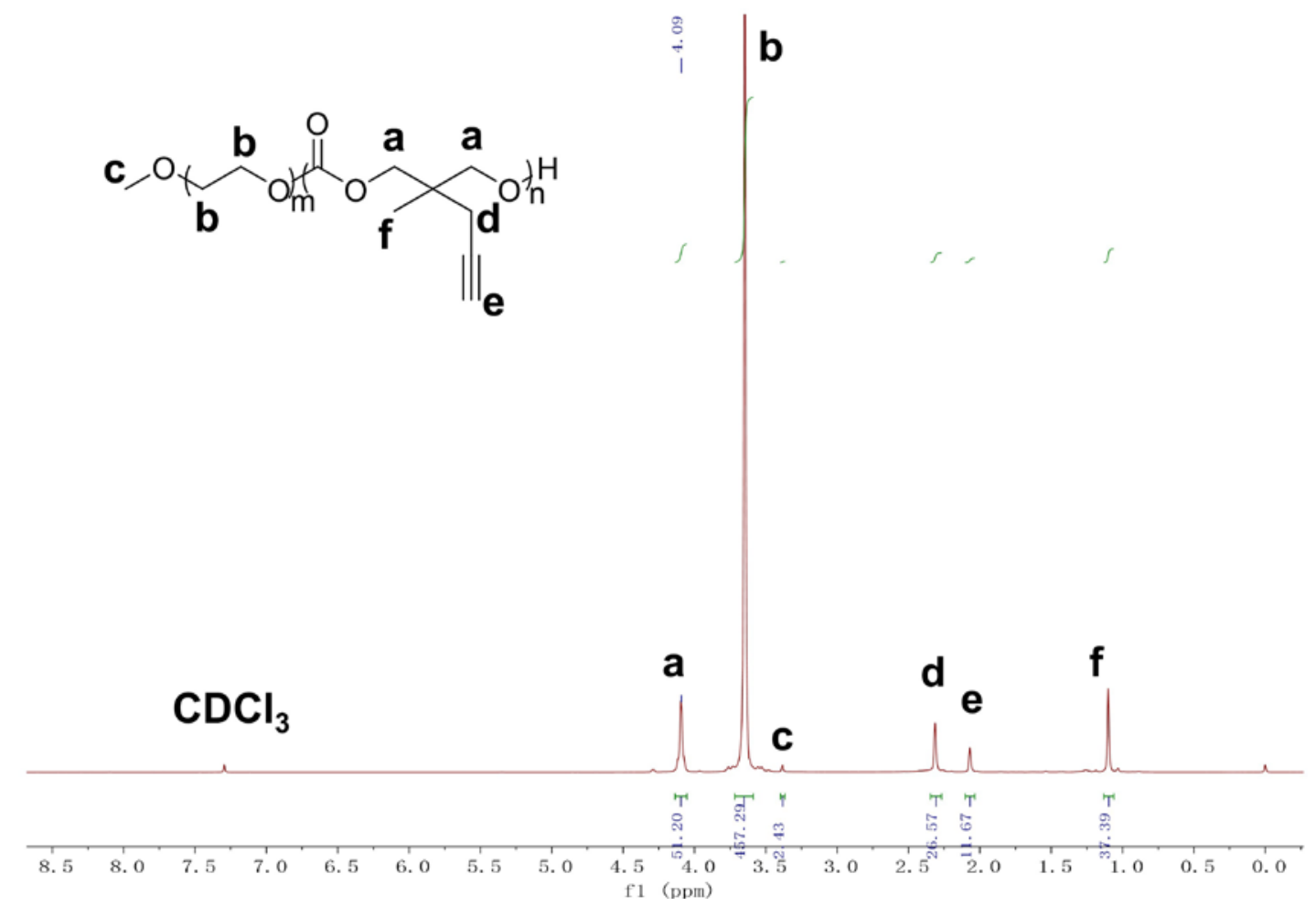

Figure S3. ${ }^{1} \mathrm{H}$ NMR spectrum $\left(400 \mathrm{MHz}, \mathrm{CDCl}_{3}\right)$ of $\mathrm{PM}$. 


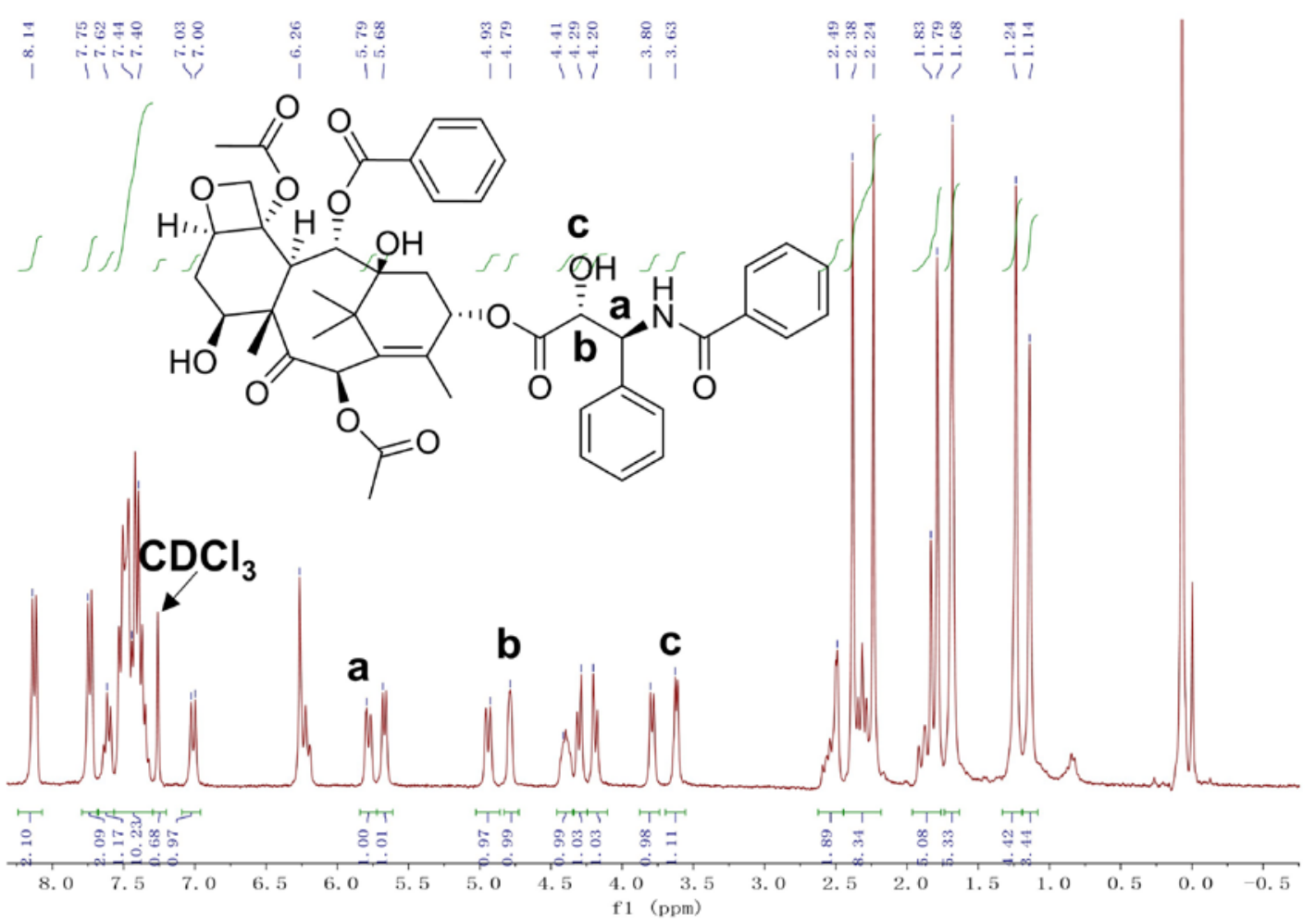

Figure S4. ${ }^{1} \mathrm{H}$ NMR spectrum (400 $\left.\mathrm{MHz}, \mathrm{CDCl}_{3}\right)$ of PTX. 


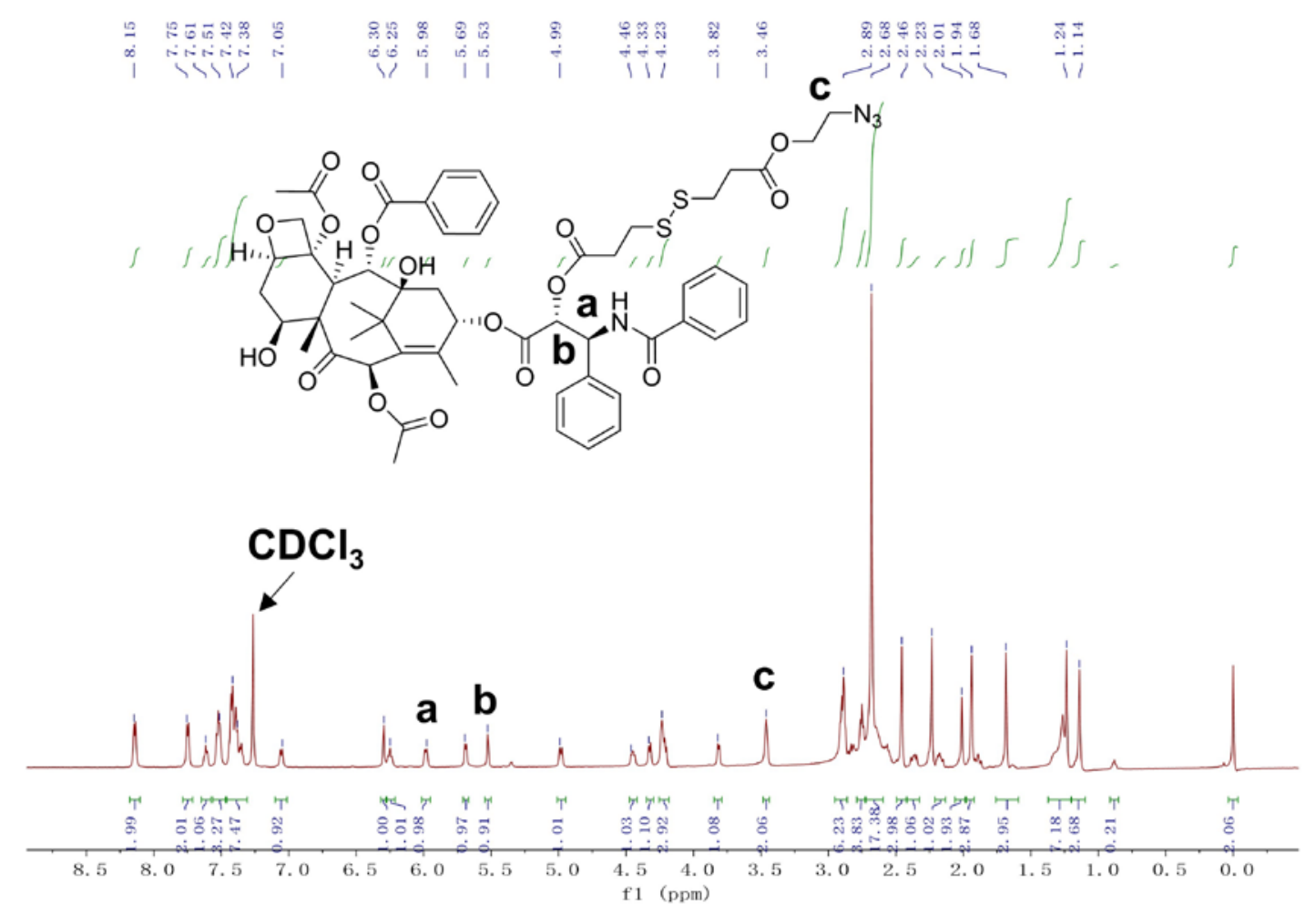

Figure S5. ${ }^{1} \mathrm{H}$ NMR spectrum $\left(400 \mathrm{MHz}, \mathrm{CDCl}_{3}\right)$ of PTX-SS-N3. 


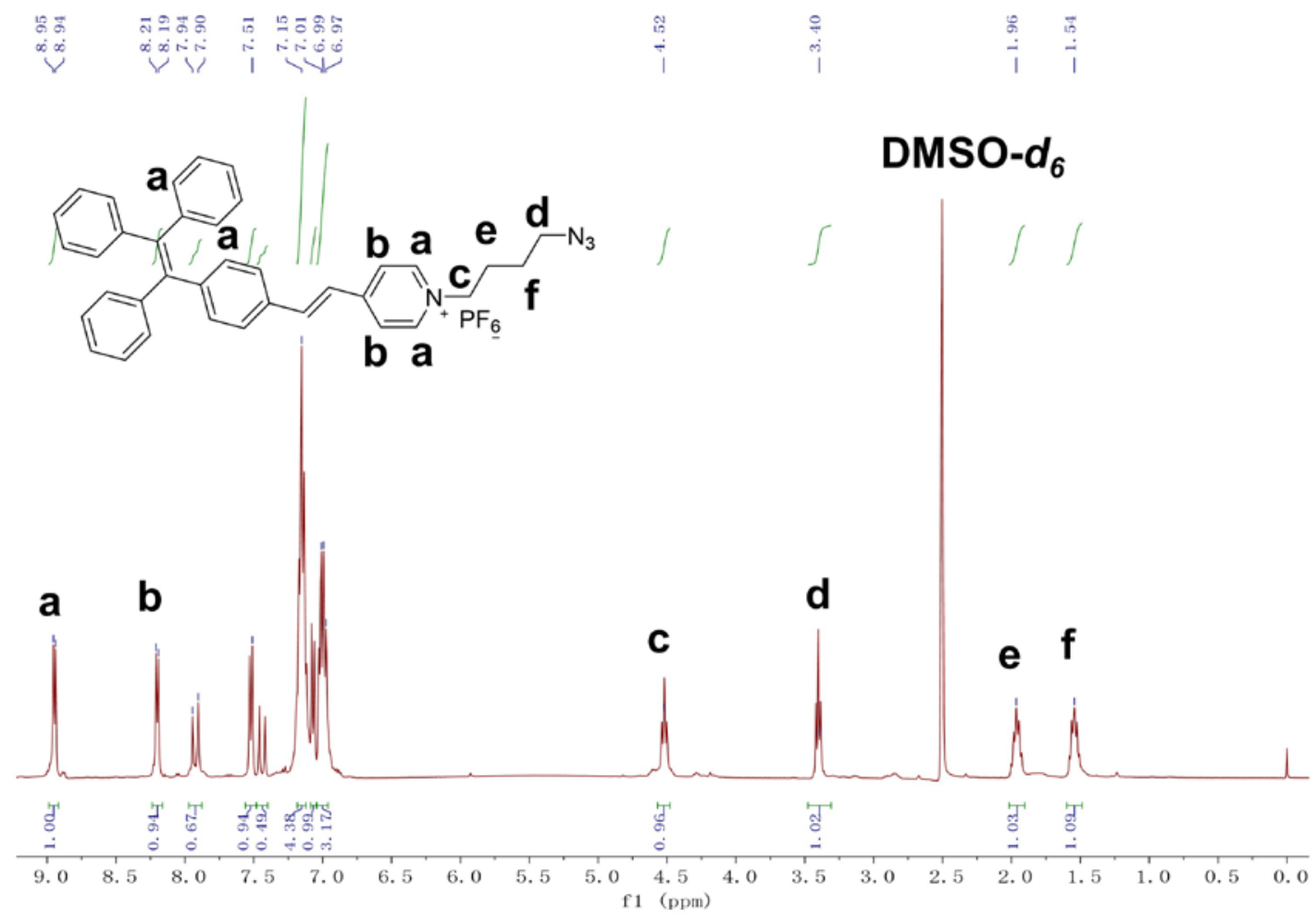

Figure S6. ${ }^{1} \mathrm{H}$ NMR spectrum (400 MHz, DMSO-d ${ }_{6}$ ) of PyTPE. 


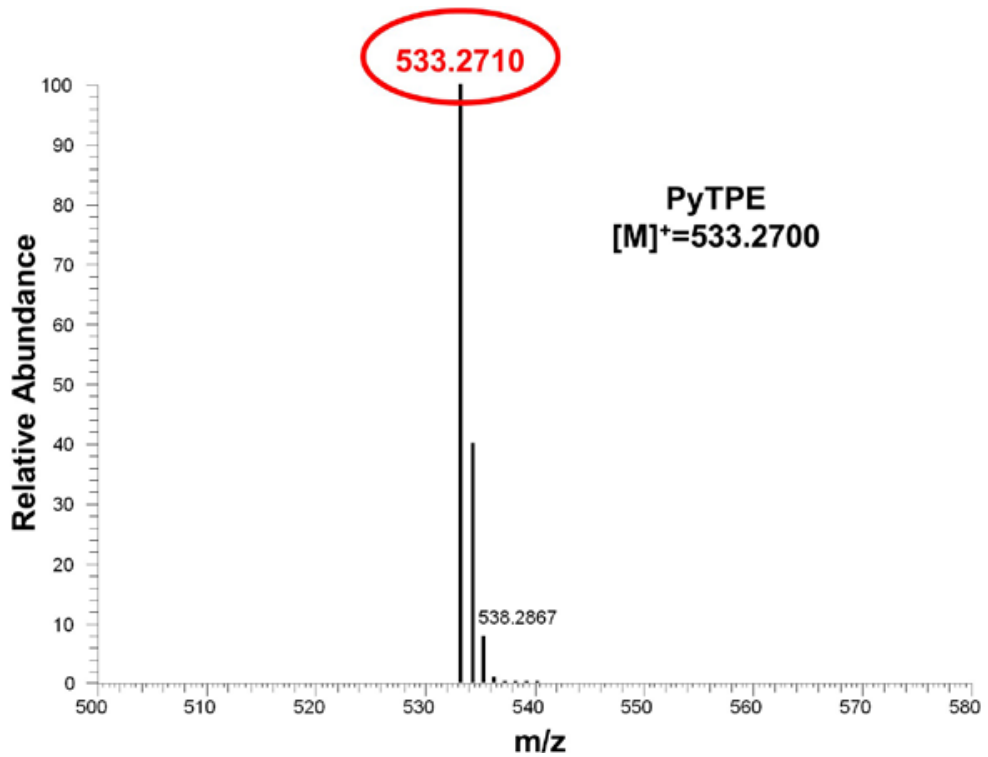

Figure S7. Mass spectrum of PyTPE, calcd [M] 553.2700. 


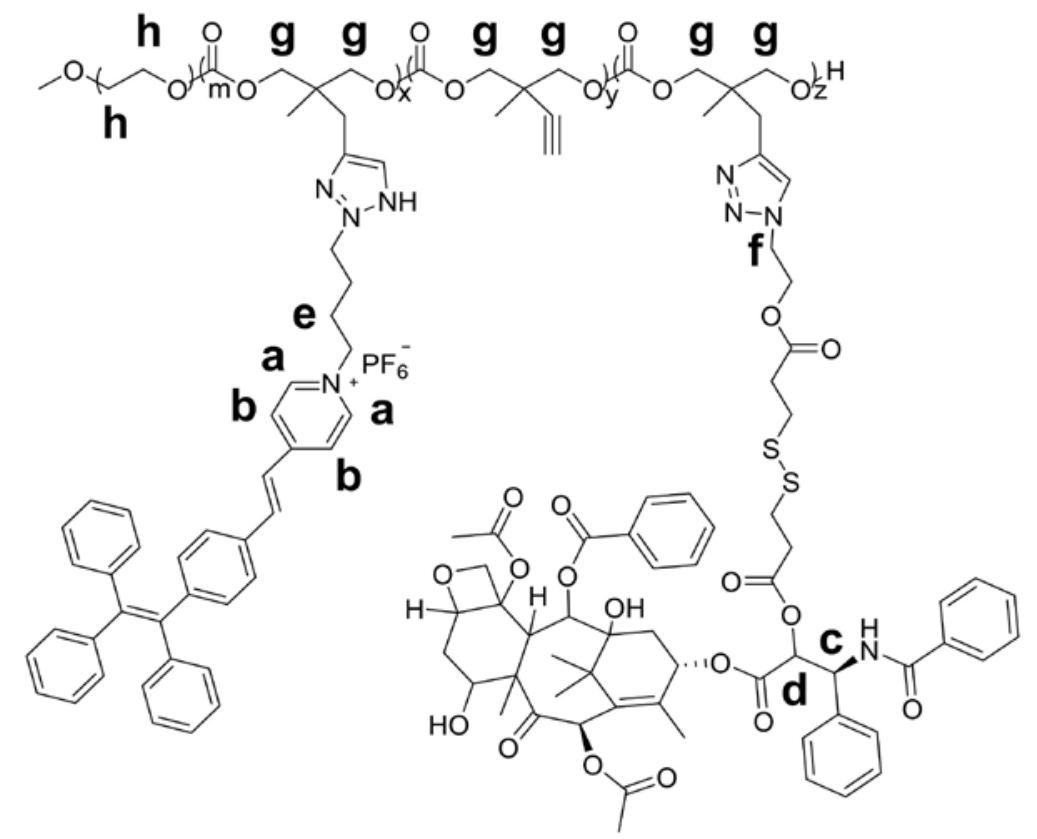

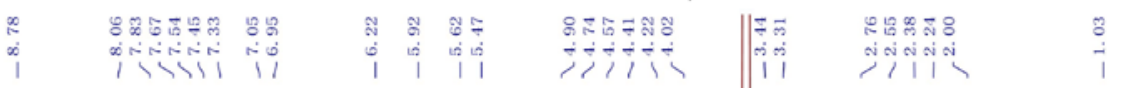

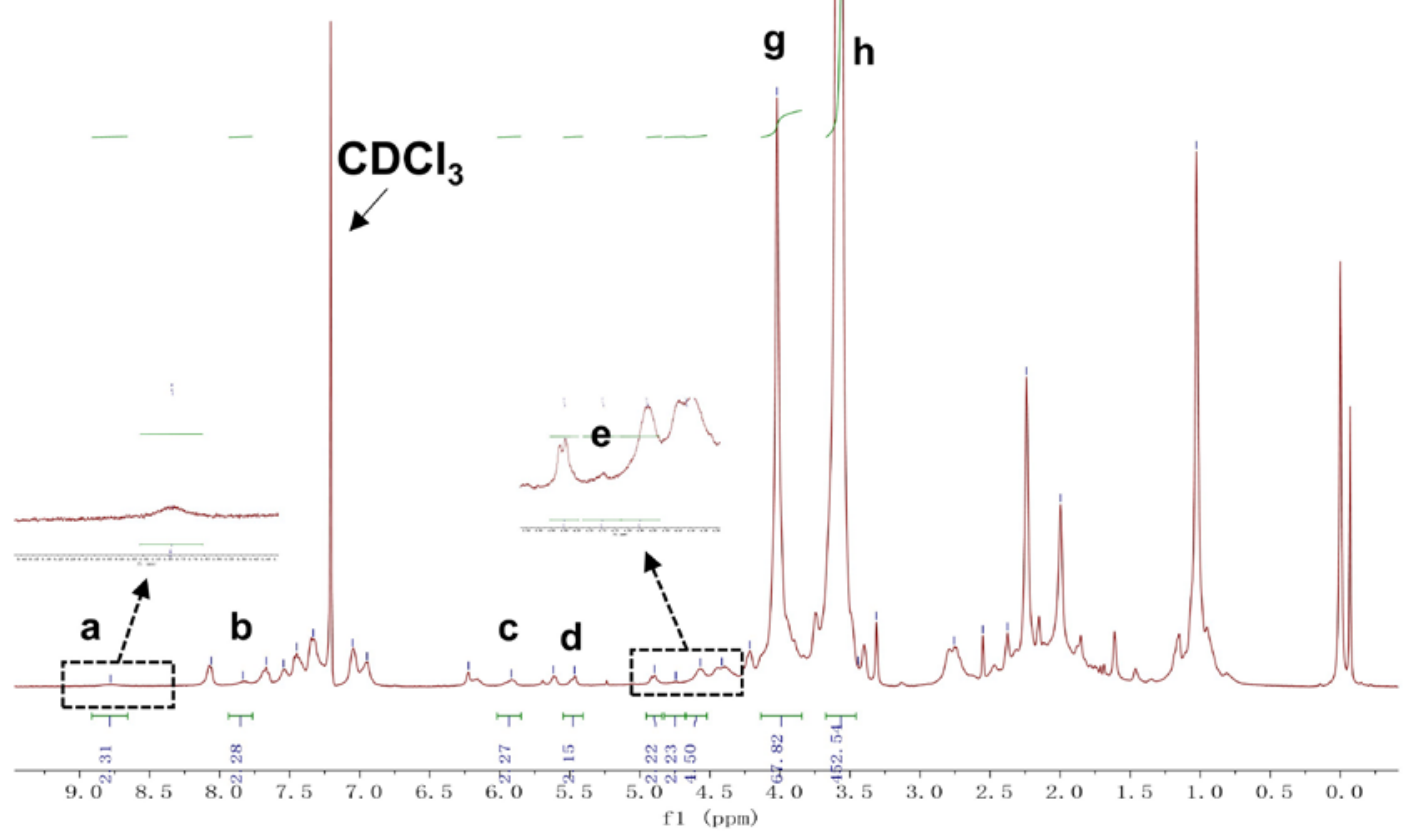

Figure S8. ${ }^{1} \mathrm{H}$ NMR spectrum $\left(400 \mathrm{MHz}, \mathrm{CDCl}_{3}\right)$ of PMPT. 


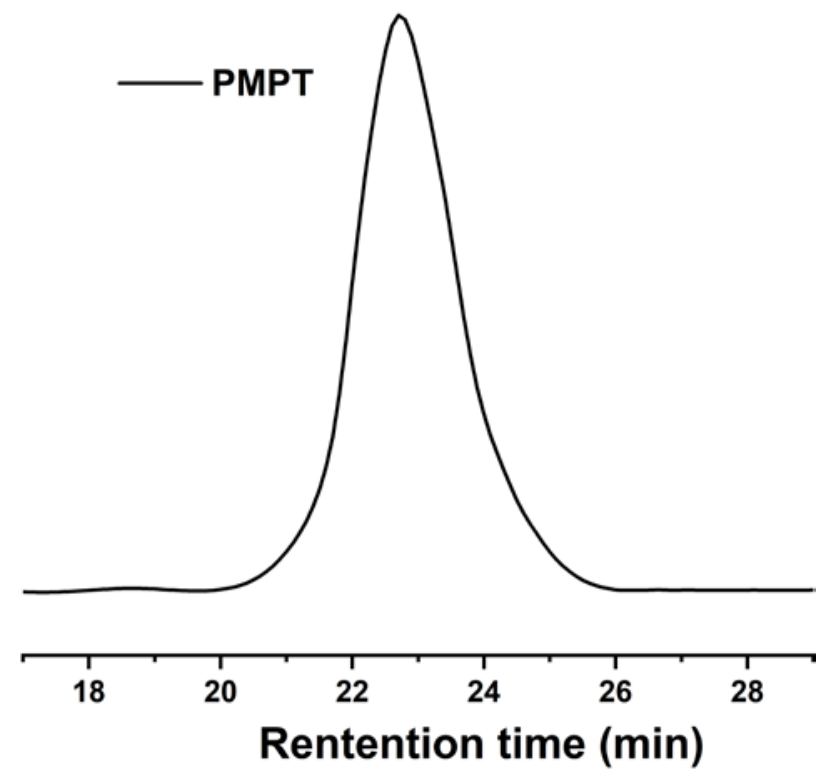

Figure S9. Gel permeation chromatography trace of PMPT. 


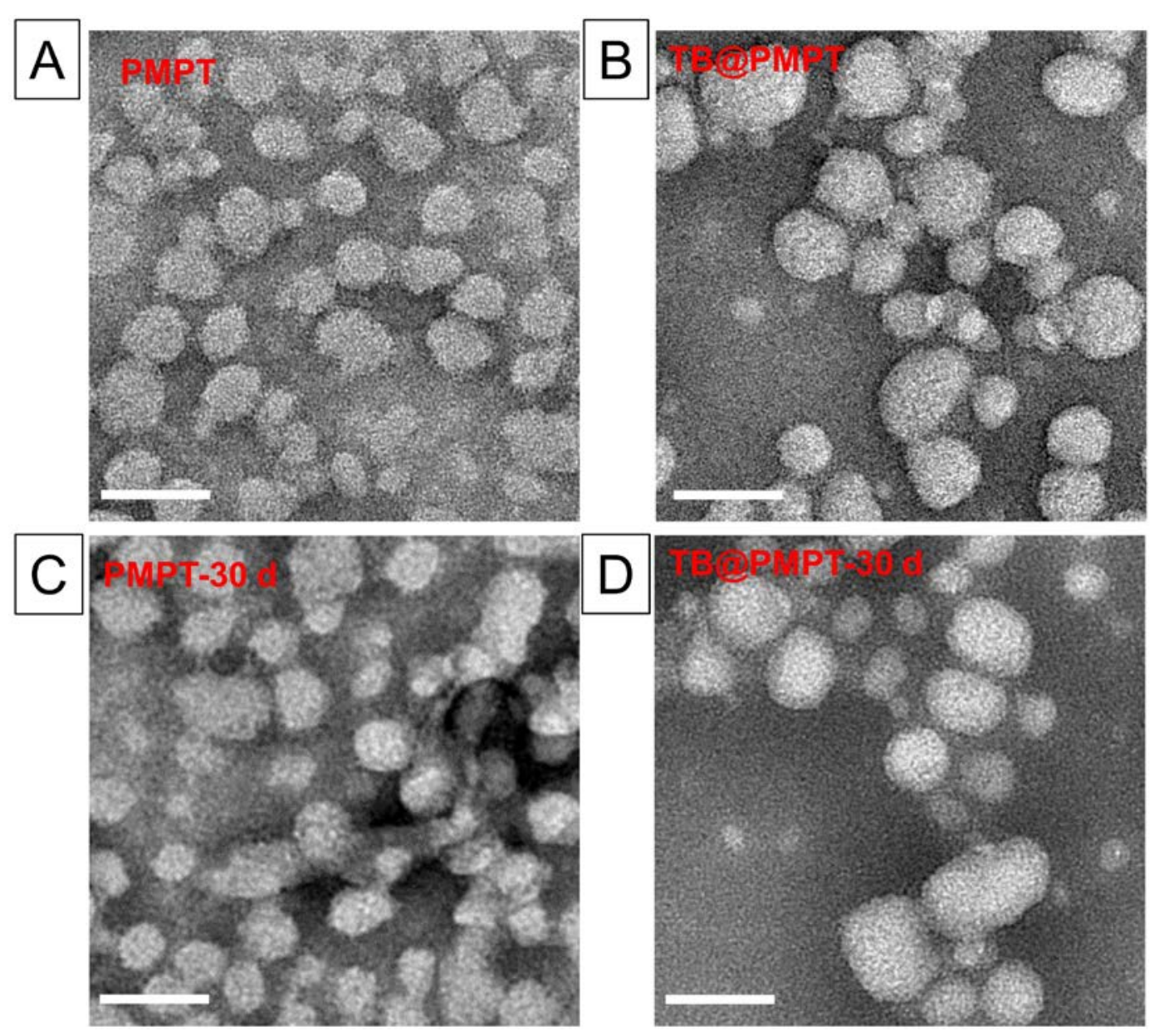

Figure S10. The transmission electron microscopy (TEM) images (A) PMPT and (B)

TB@PMPT micelles with TB loading of 9.57\%. The TEM images of (C) PMPT and

(D) TB@PMPTmicelles with TB loading of 9.57\% after stored at room temperature for

30 days. The scale bar is $100 \mathrm{~nm}$. 

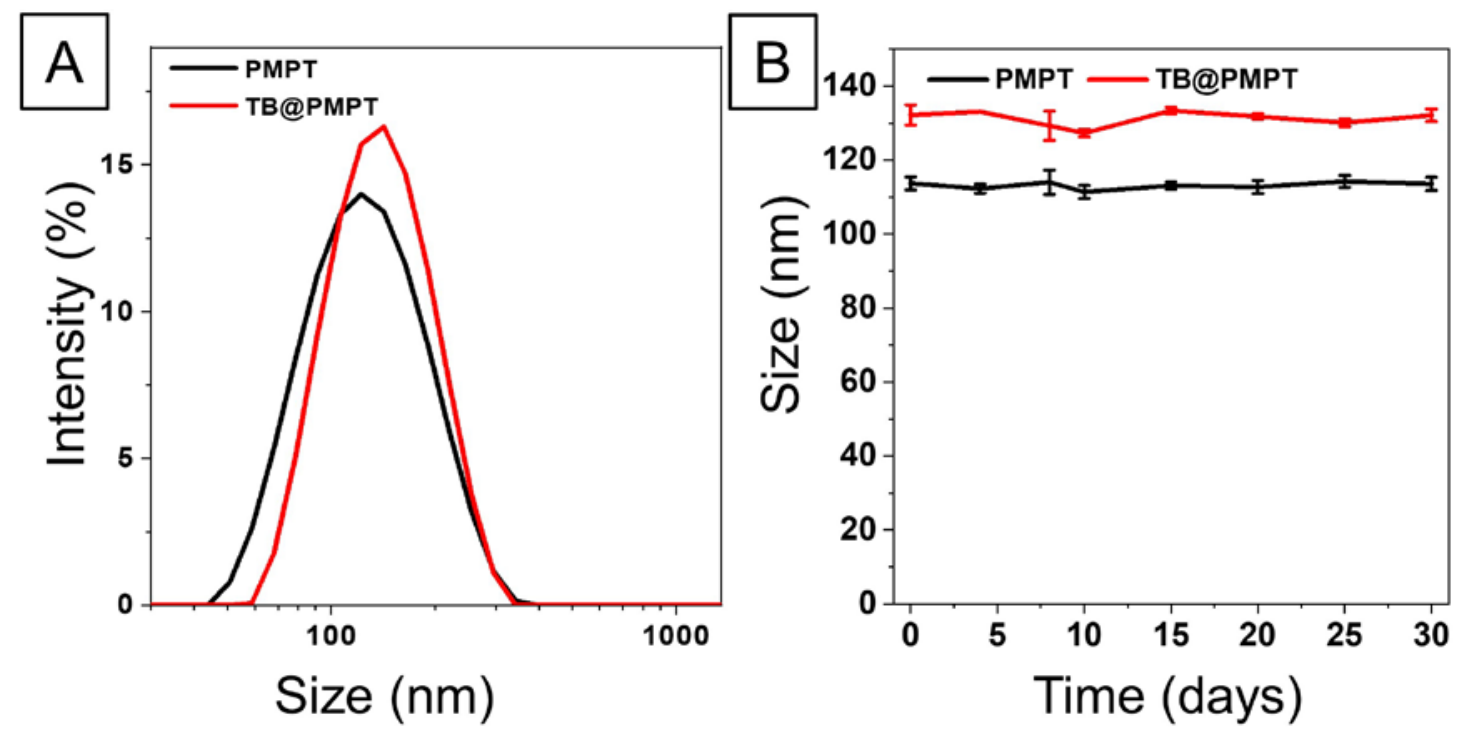

Figure S11. (A) Hydrodynamic size distribution of PMPT and TB@PMPT micelles in PBS. (B) Stability assay of PMPT and TB@PMPT micelles in PBS during 30-day storage at room temperature. 


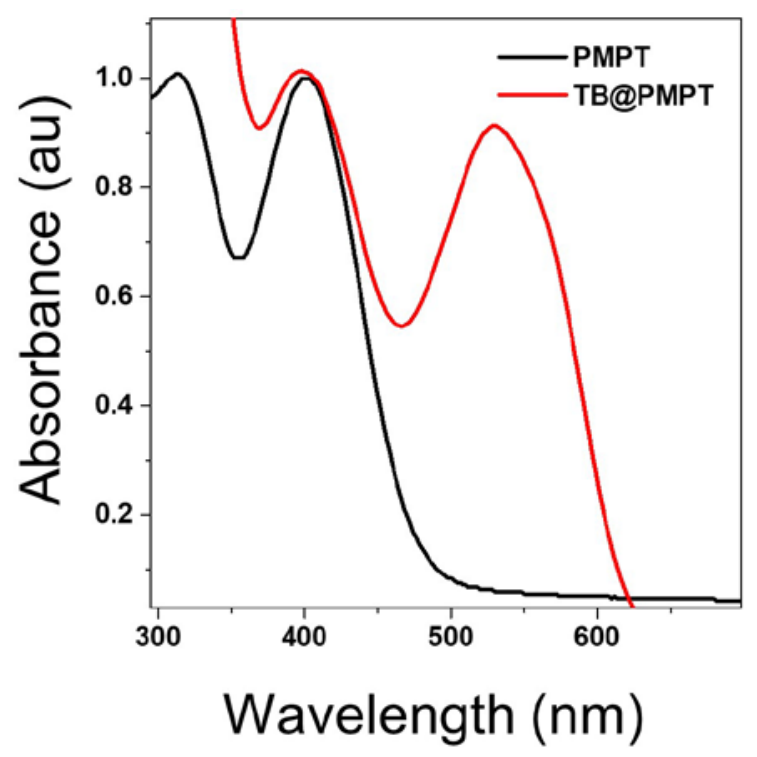

Figure S12. UV-vis spectrum of (A) PMPT and (B) TB@PMPT micelles. 

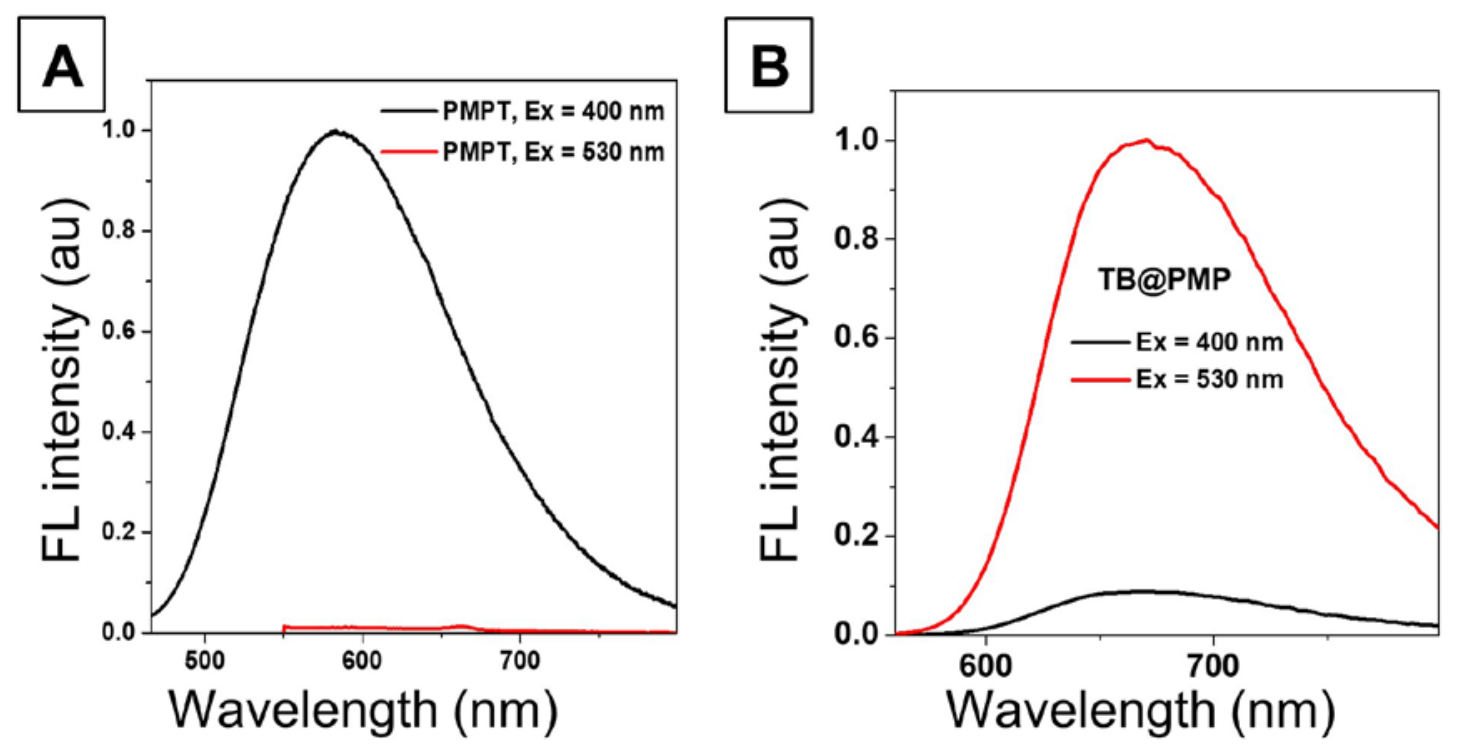

Figure S13. Fluorescence spectra (Ex: 400 and $530 \mathrm{~nm}$ ) of (A) PMPT and (B) TB@PMP micelles in aqueous solution. 

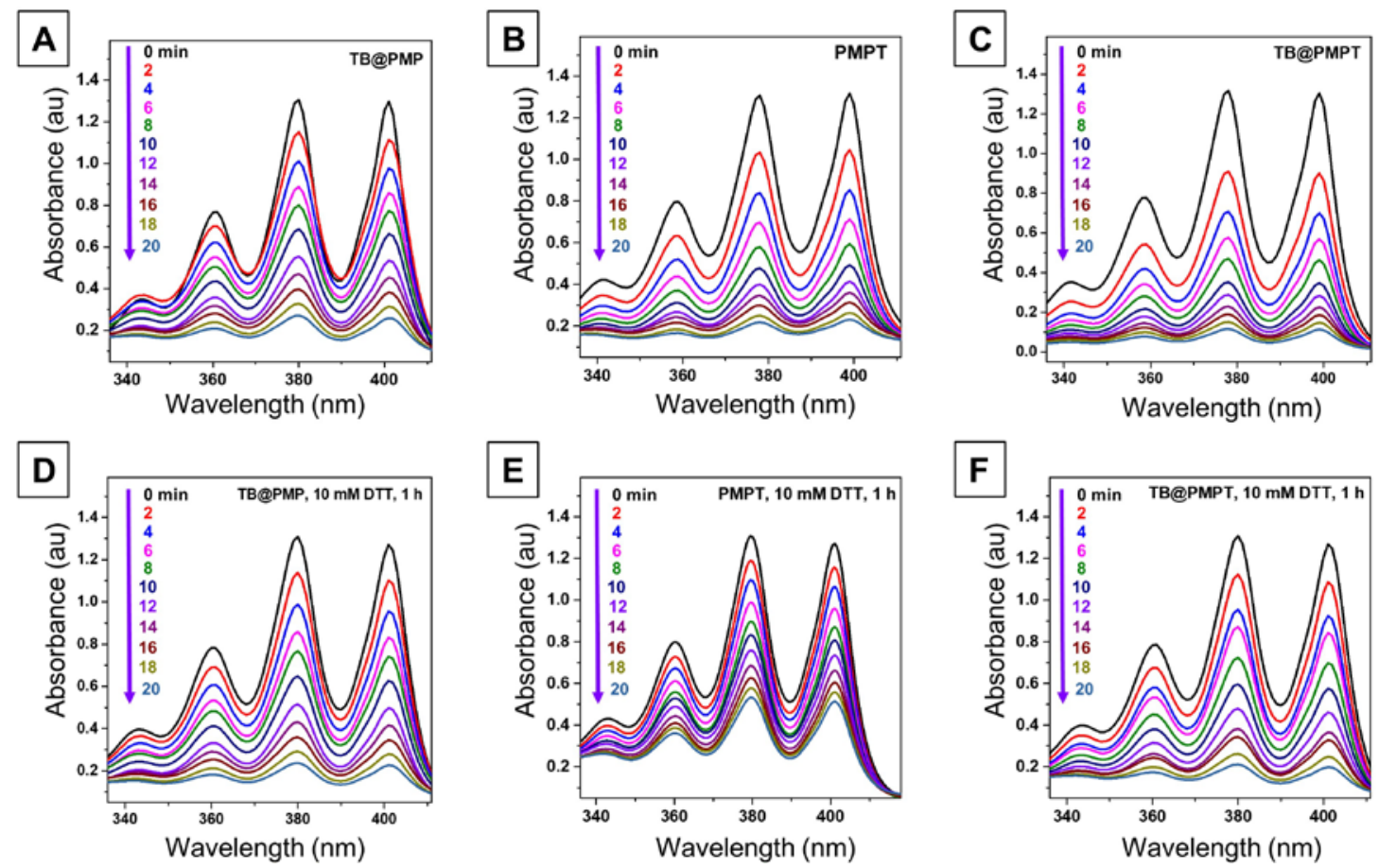

Figure S14. UV-vis absorption spectra of the ROS indicator 9,10anthracenediylbis(methylene)dimalonic acid (ABDA) mixed with various micelles upon light irradiation (white light, $70 \mathrm{~mW} \mathrm{~cm} \mathrm{~cm}^{-2}$ ). (A) TB@PMP; (B) PMPT; (C) TB@PMPT; (D) TB@PMP micelles with 10 mM DTT were pre-incubated at $37^{\circ} \mathrm{C}$ for $1 \mathrm{~h}$; (E) PMPT micelles with $10 \mathrm{mM}$ DTT were pre-incubated at $37^{\circ} \mathrm{C}$ for $1 \mathrm{~h}$; (F) TB@PMPT micelles with $10 \mathrm{mM}$ DTT were pre-incubated at $37^{\circ} \mathrm{C}$ for $1 \mathrm{~h}$. 


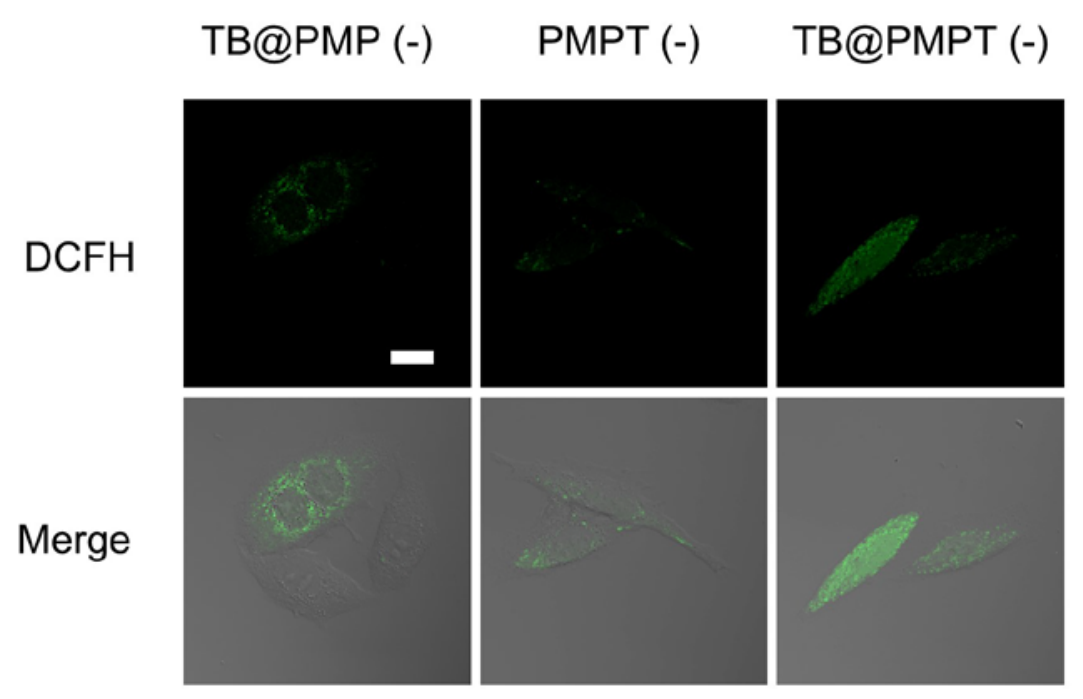

Figure S15. Detection of intracellular ROS production by DCFH-DA in HeLa cells after incubation with TB@PMP (-), PMPT (-) and TB@PMPT (-) micelles, respectively. Scale bar: $20 \mu \mathrm{m}$. 


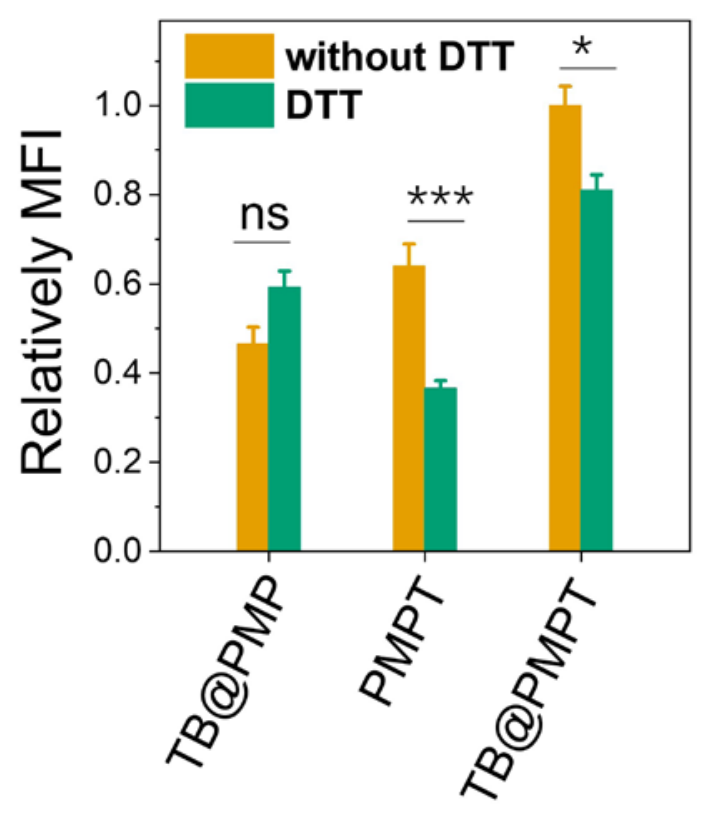

Figure S16. Corresponding quantitative analysis of the fluorescence intensity in Figure 1B by the Zeiss LSM 880 software. Data was reported as mean \pm SD and analysed through two-sided Student's $t$-test. $\left({ }^{* *} p<0.001,{ }^{*} p<0.05\right.$, ns means not significant). 


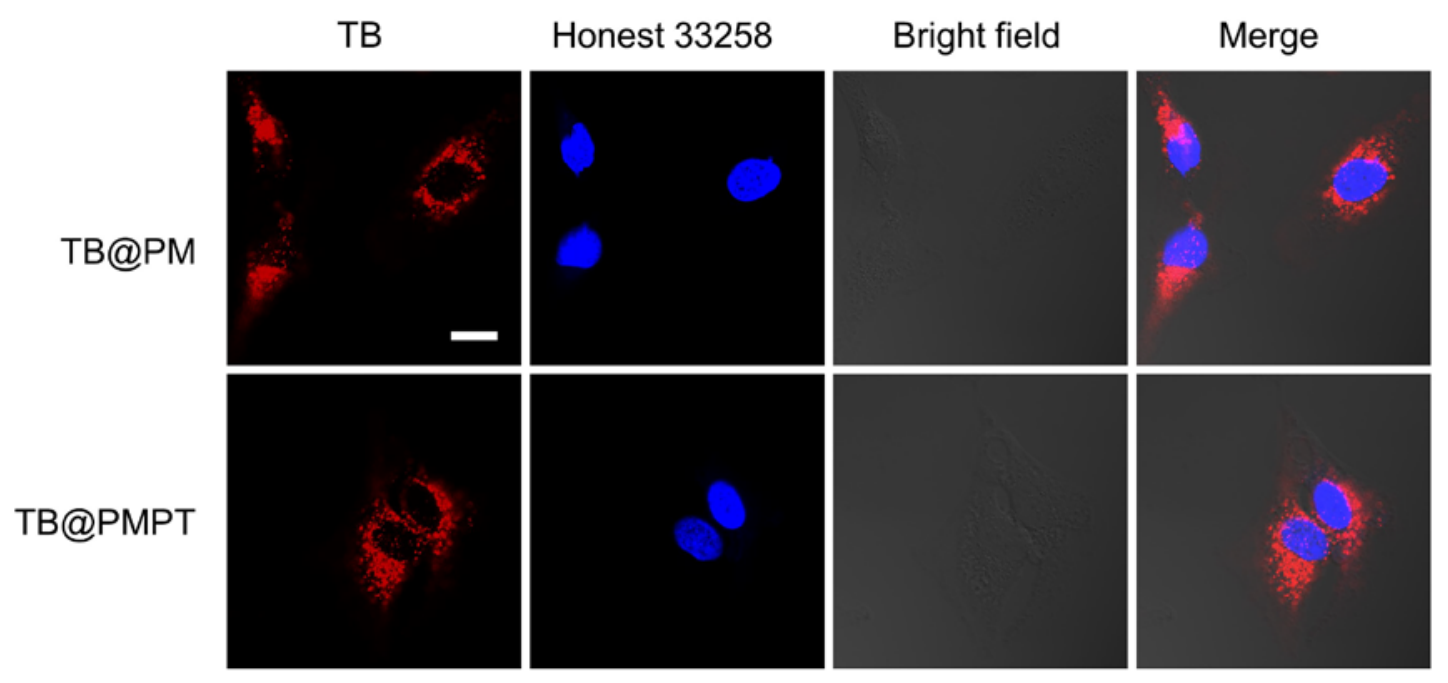

Figure S17. CLSM images of HeLa cells after incubation with TB@PM and TB@PMPT micelles for $4 \mathrm{~h}$. Scale bar is $20 \mu \mathrm{m}$. 

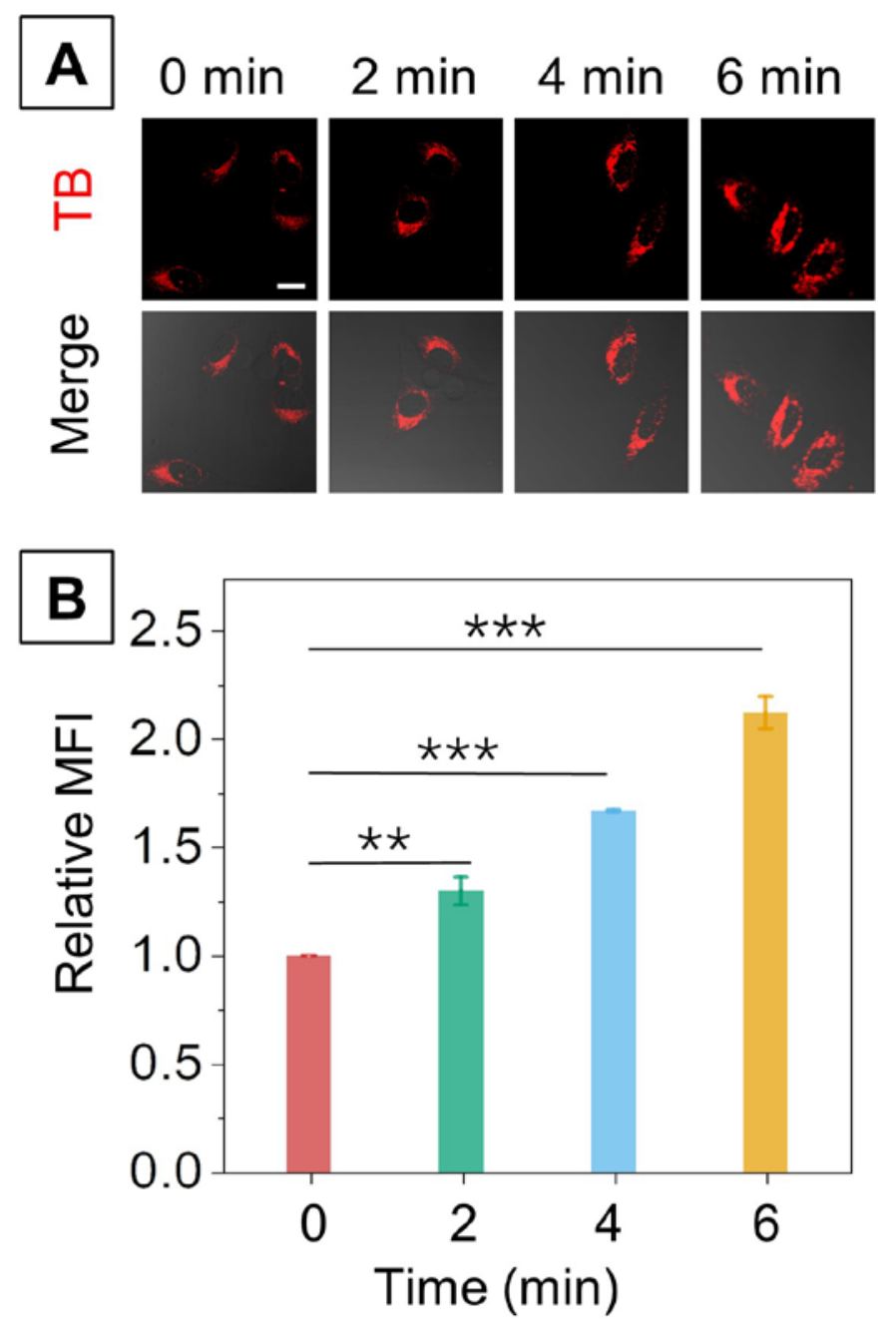

Figure S18. (A) CLSM images of HeLa cells after incubation with TB@PMPT micelles with the first light irradiation $\left(\mathrm{L}_{1}\right)$ for $0 \mathrm{~min}, 2 \mathrm{~min}, 4 \mathrm{~min}$ and $6 \mathrm{~min}$, respectively. (B) Corresponding quantitative analysis of the releative fluorescence intensity of TB in CLSM images by the Zeiss LSM 880 software. 


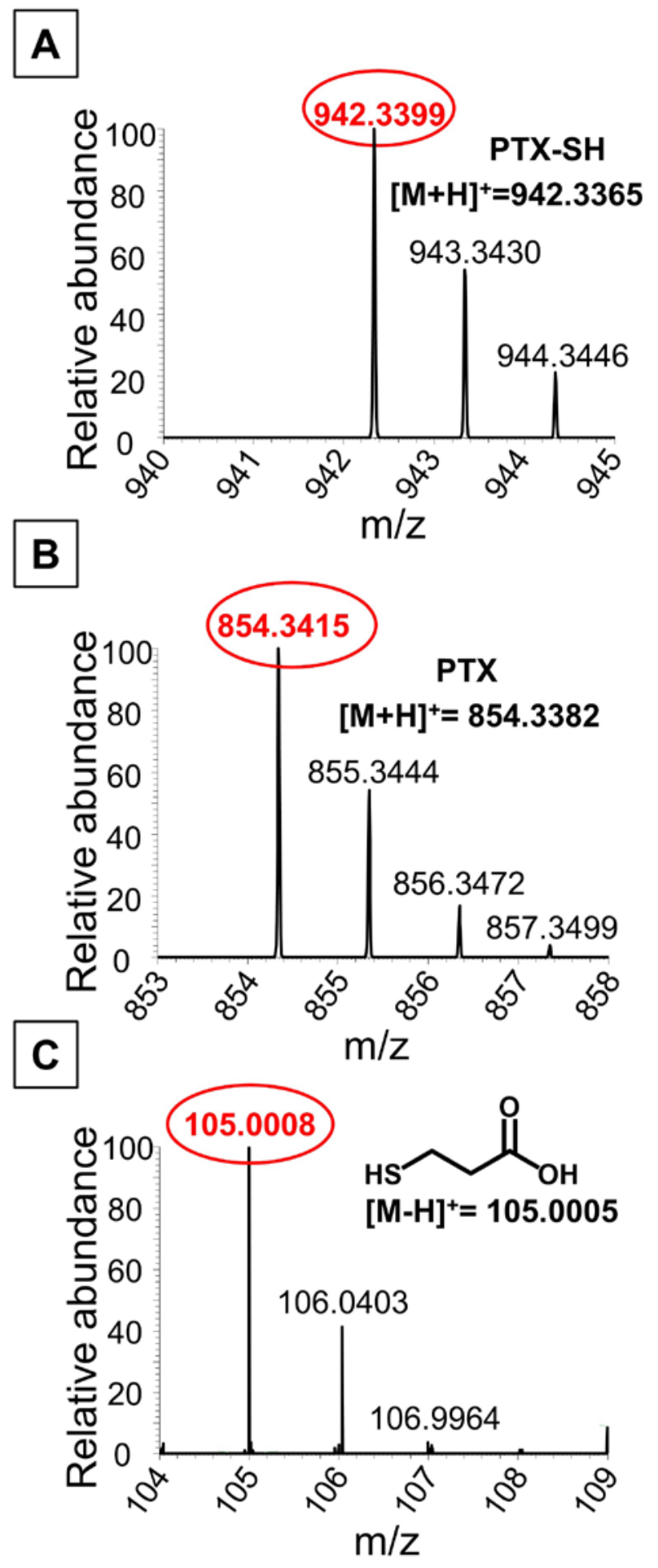

Figure S19. Mass spectra of the released products from PTX-SS-N $\mathrm{N}_{3}$ with the treatment of 10 mM DTT. (A) PTX-SH: [M+H] 942.3399 (calcd 942.3365); (B) (PTX): [M+H] 854.3415 (calcd 854.3382); (C) 3-mercaptopropanoic acid: [M-H] 105.0008 (calcd 105.0005). 


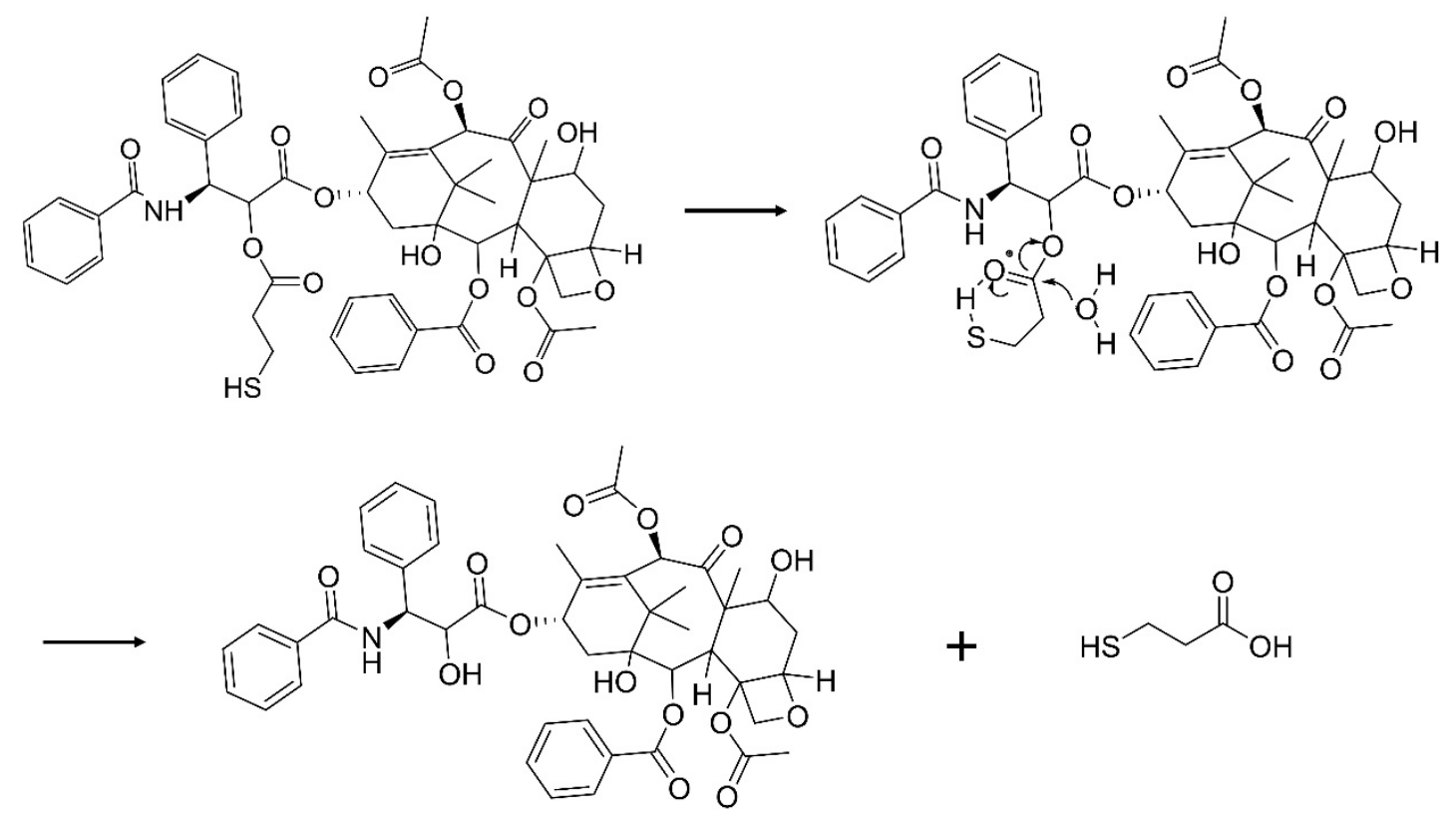

Figure S20. The release mechanism of free PTX from PTX-SH under the condition of $10 \mathrm{mM}$ DTT. 

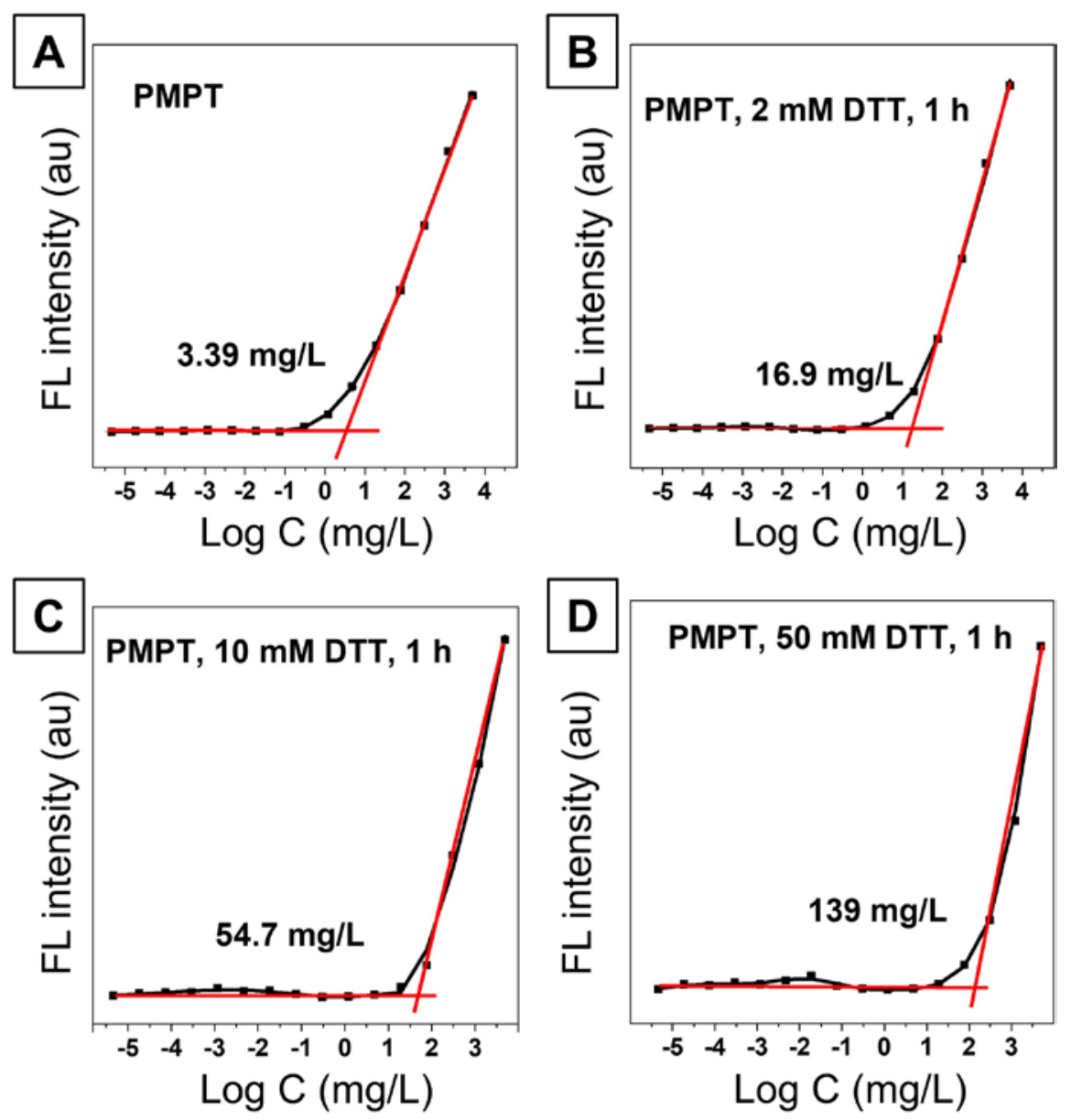

Figure S21. The critical micelle concentration (CMC) value after PMPT micelles incubated with different concentrations of DTT for 1 h: (A) 0 mM DTT; (B) 2 mM DTT; (C) 10 mM DTT; (D) 50 mM DTT. 

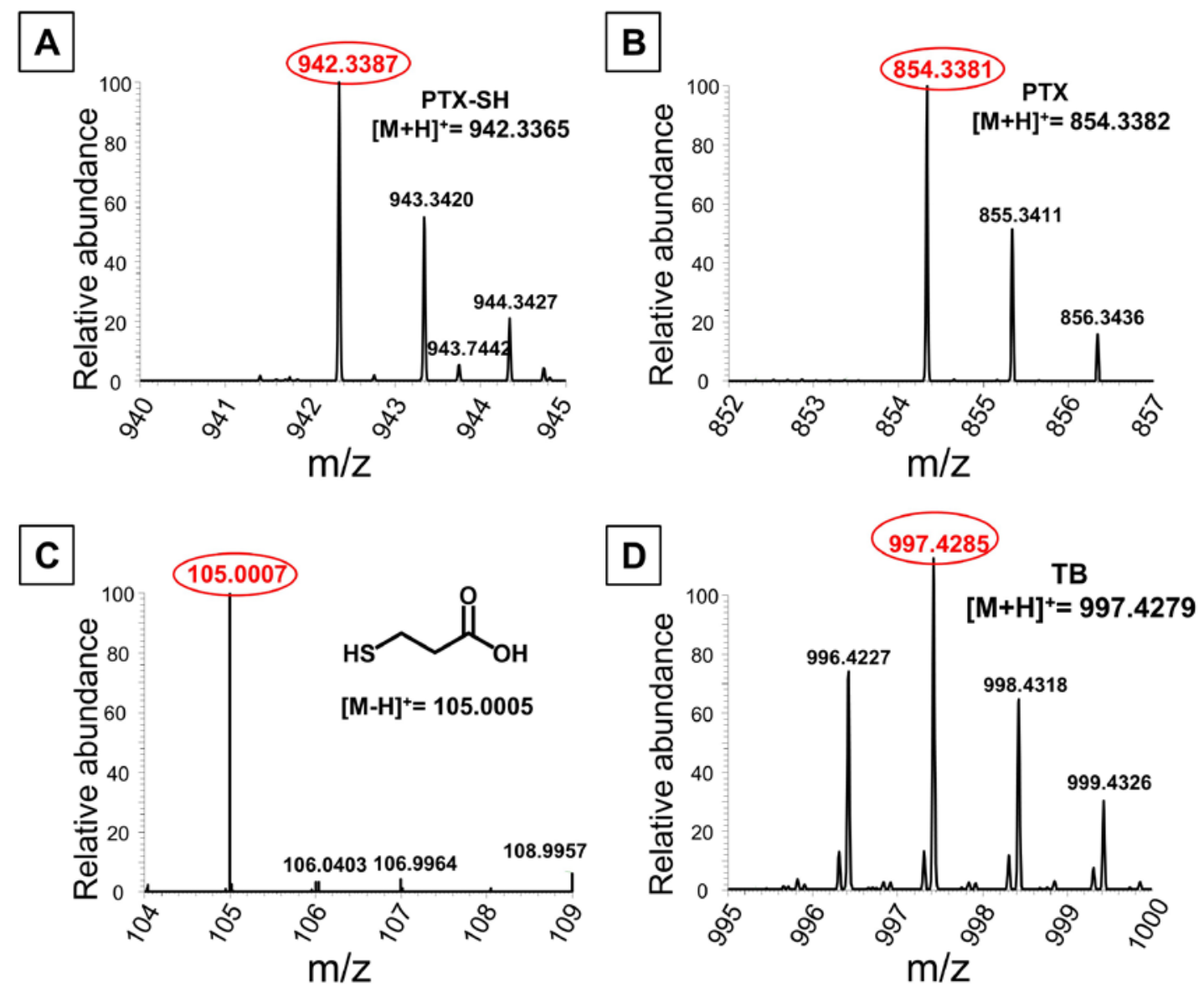

Figure S22. Mass spectra of the released products from TB@PMPT micelles with the treatment of 10 mM DTT. HRMS (A) PTX-SH: [M+H] 942.3387 (calcd 942.3365); (B) (PTX): [M+H] 854.3381 (calcd 854.3382); (C) 3-mercaptopropanoic acid: [M-H] ${ }^{+}$ 105.0007 (calcd 105.0005); (D) TB: [M+H]+ 997.4285 (calcd 997.4279). 

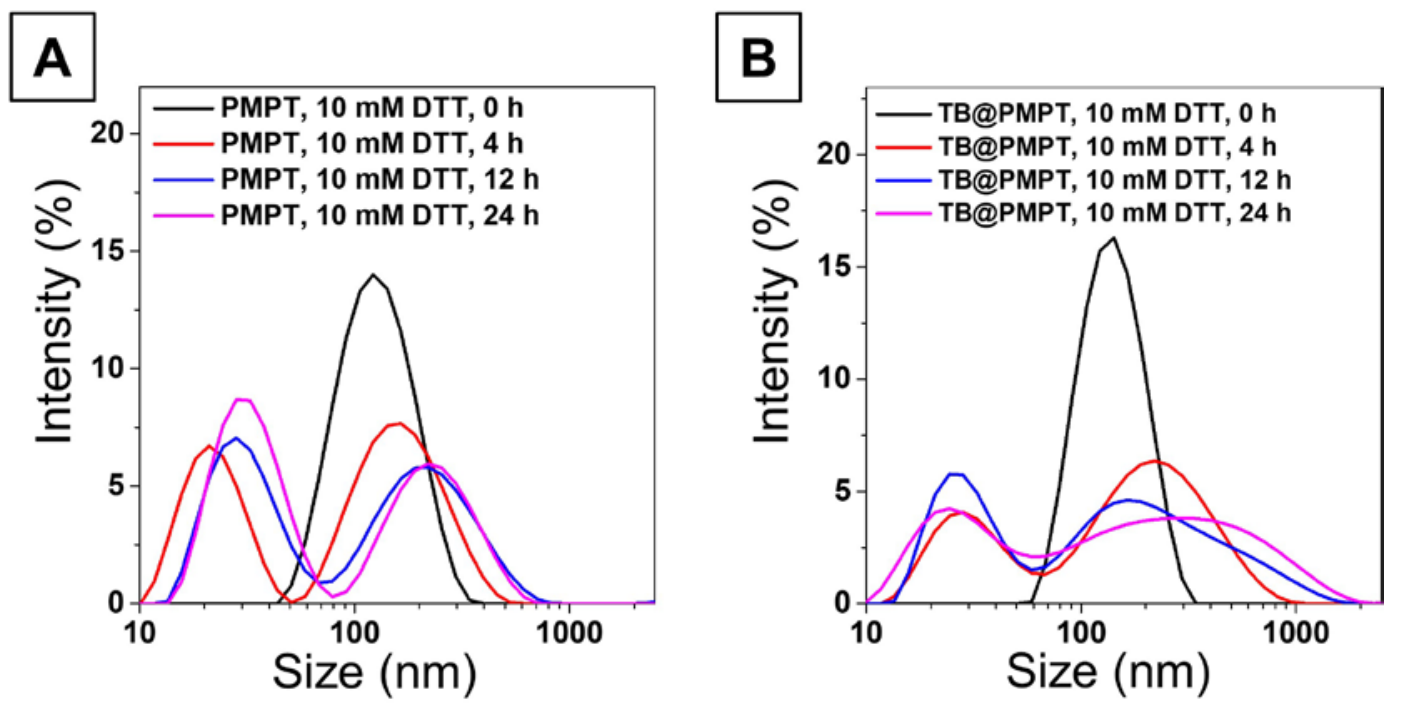

Figure S23. The size changes of (A) PMPT and (B) TB@PMPT micelles with TB loading of $9.57 \%$ in response to $10 \mathrm{mM}$ DTT at $\mathrm{pH} 7.4$ determined by DLS, respectively. 

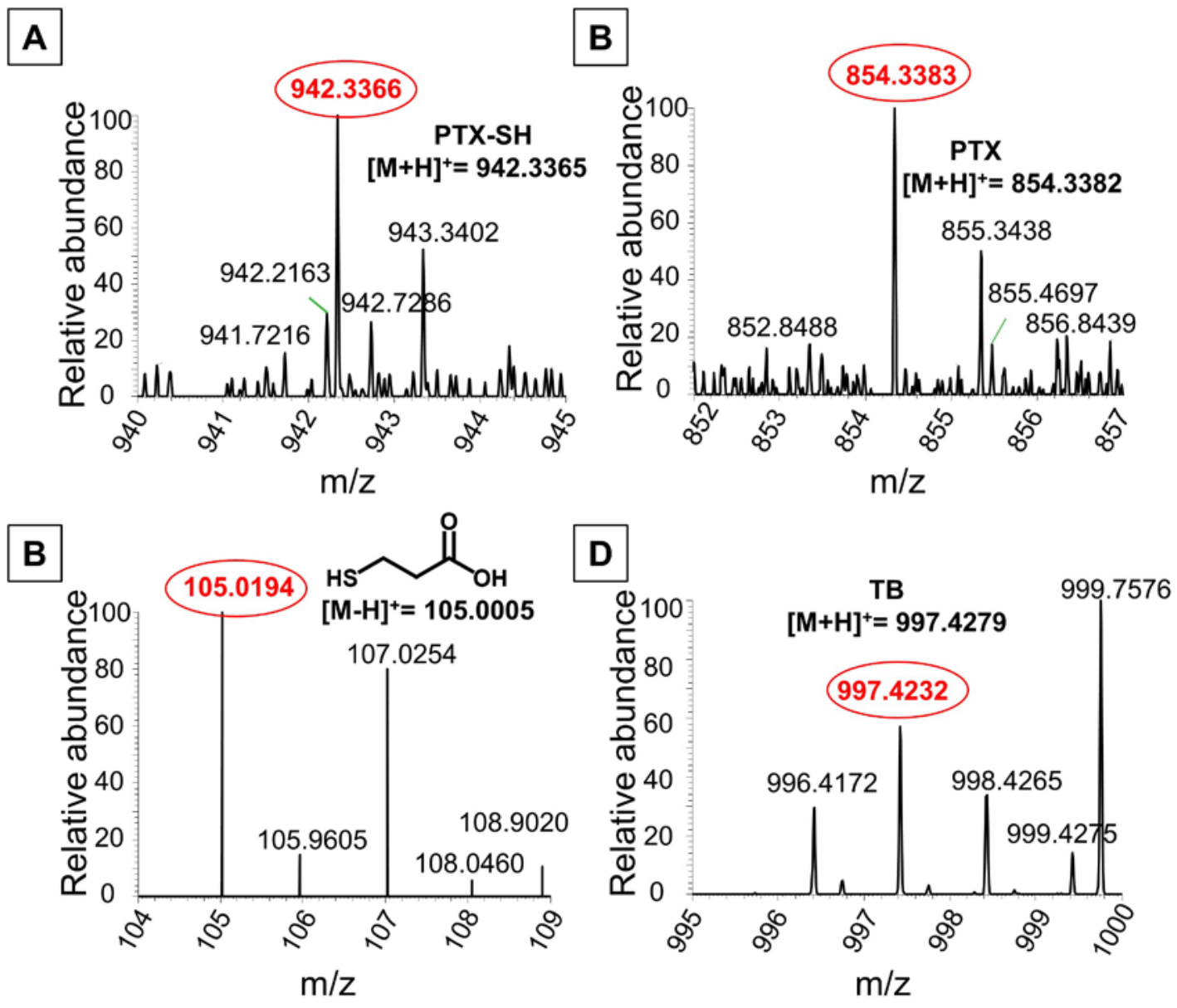

Figure S24. Mass spectra of the cell extract after incubation of TB@PMPT micelles with HeLa cells. HRMS (A) PTX-SH: [M+H] 942.3366 (calcd 942.3365); (B) (PTX): $[\mathrm{M}+\mathrm{H}]^{+} 854.3383$ (calcd 854.3382); (C) 3-mercaptopropanoic acid: [M-H] 105.0194 (calcd 105.0005); (D) TB: [M+H]+ 997.4232 (calcd 997.4279). 

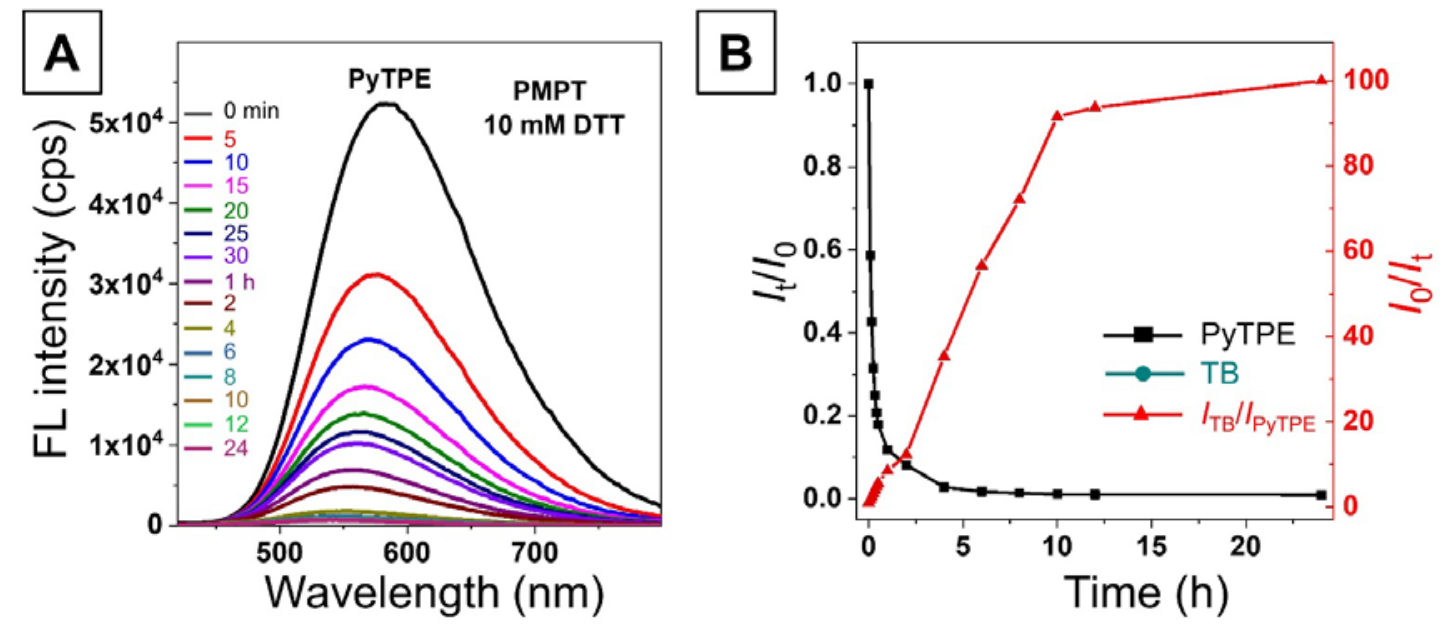

Figure S25. (A) Fluorescence (FL) spectra, (B) fluorescence intensity ratios $I_{t} / I_{0}$ and fluorescence intensity ratios of $I_{0} / I_{\mathrm{t}}$ of PMPT micelles $\left(100 \mathrm{mg} \mathrm{L}^{-1}\right)$ was incubated with $10 \mathrm{mM}$ DTT for different times. Ex $=400 \mathrm{~nm}, \mathrm{Em}=583 \mathrm{~nm}$. 

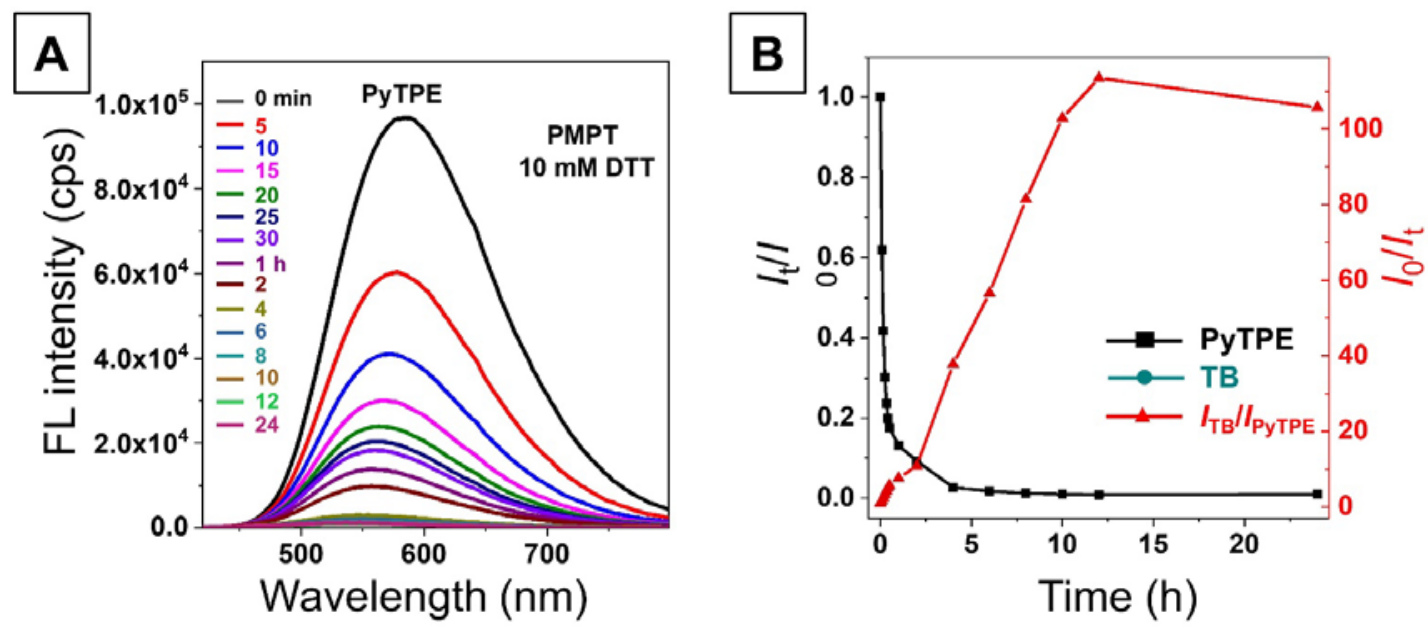

Figure S26. (A) FL spectra, (B) fluorescence intensity ratios $I t / I_{0}$ and fluorescence intensity ratios of $I_{0} / I_{\mathrm{t}}$ of PMPT micelles (200 $\left.\mathrm{mg} \mathrm{L}^{-1}\right)$ was incubated with $10 \mathrm{mM}$ DTT for different times. 

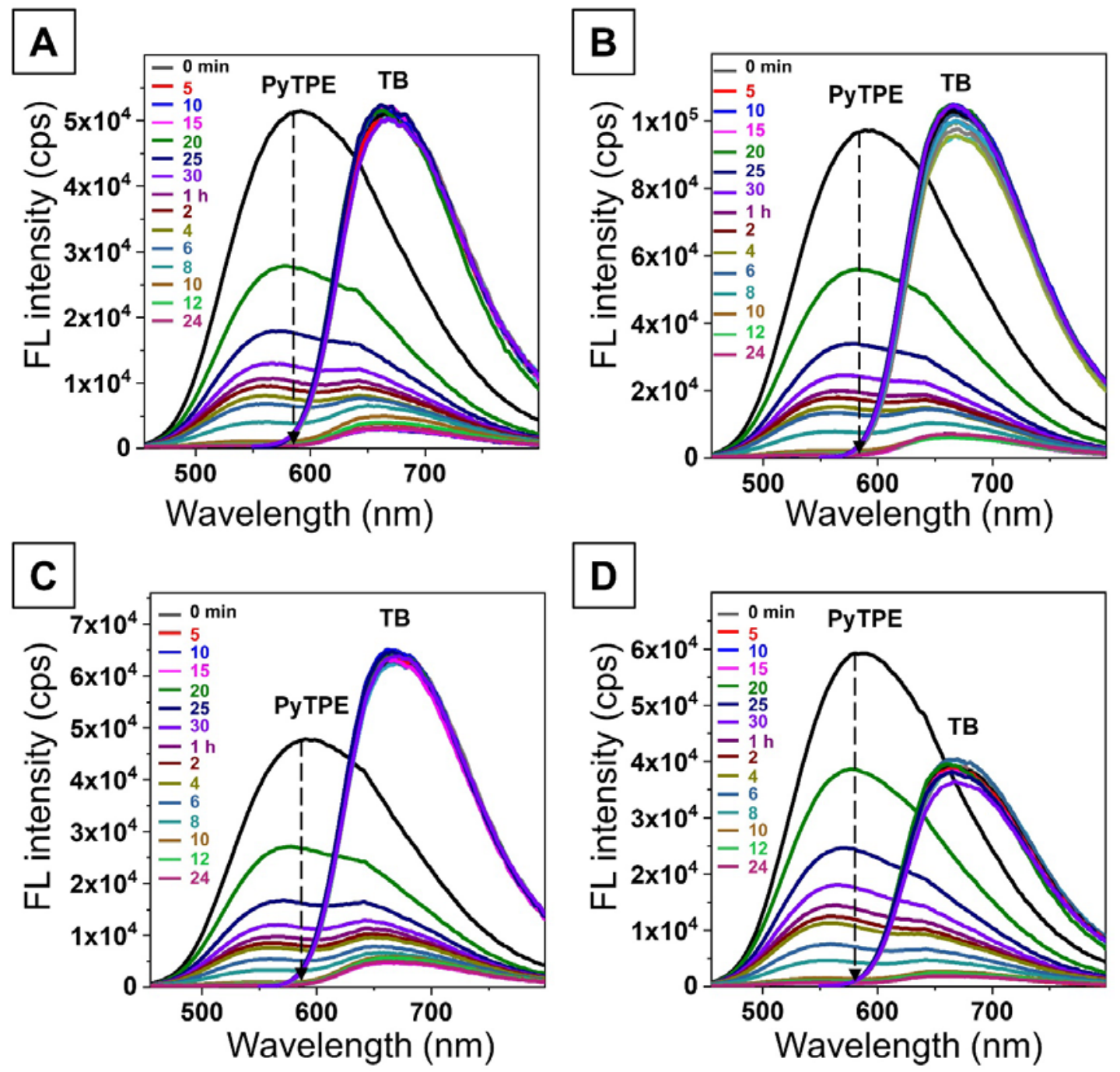

Figure S27. FL spectra of TB@PMPT micelles with different concentrations and different TB loading content incubated with 10 mM DTT for different times. (PyTPE: $\mathrm{Ex}=400$ nm, Em = 583 nm; TB: Ex $=530$ nm, Em = 684 nm). (А) СтВ@РмРт: 100 mg L-1, DLCтв: 7.57\%; (В) Ств@РмРт: 200 mg L-1, DLСтв: 7.57\%; (С) Ств@РмРт: 100 mg L-1, DLCтв: 9.57\%; (D) С тв@РмPт: 100 mg L-1, DLCтв: 10.8\%. 
A The HeLa cells without pretreatment

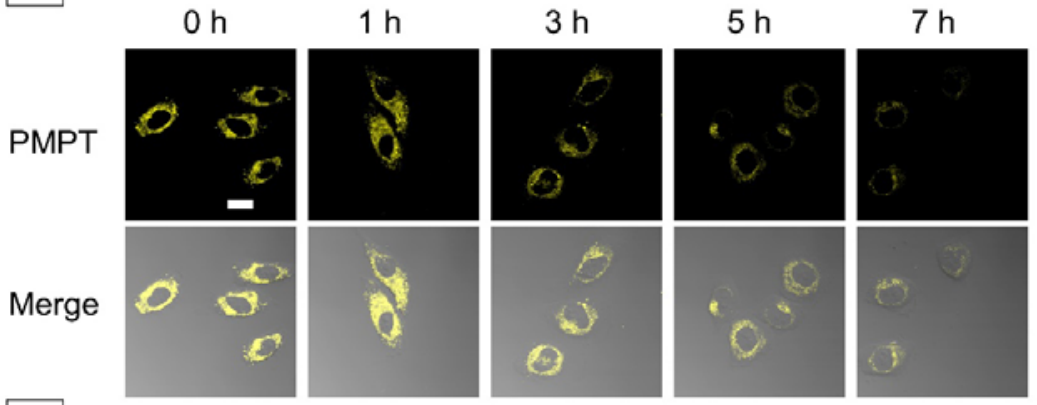

B The HeLa cells were pretreated with GSH-OEt

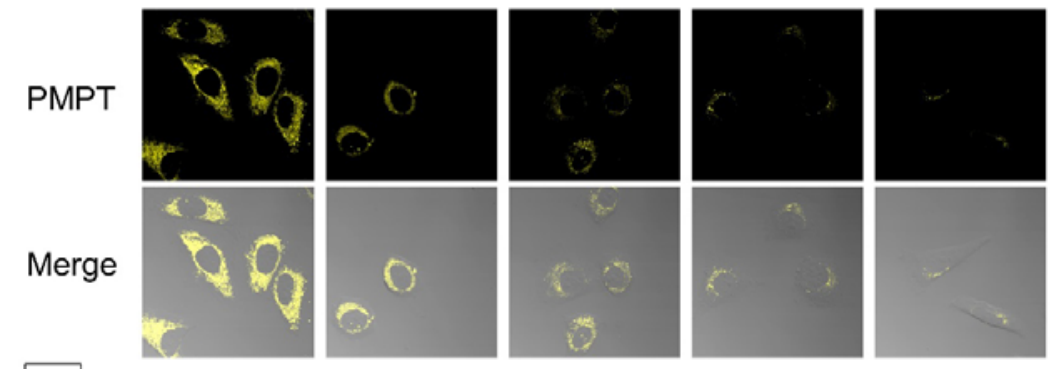

c The HeLa cells were pretreated with BSO

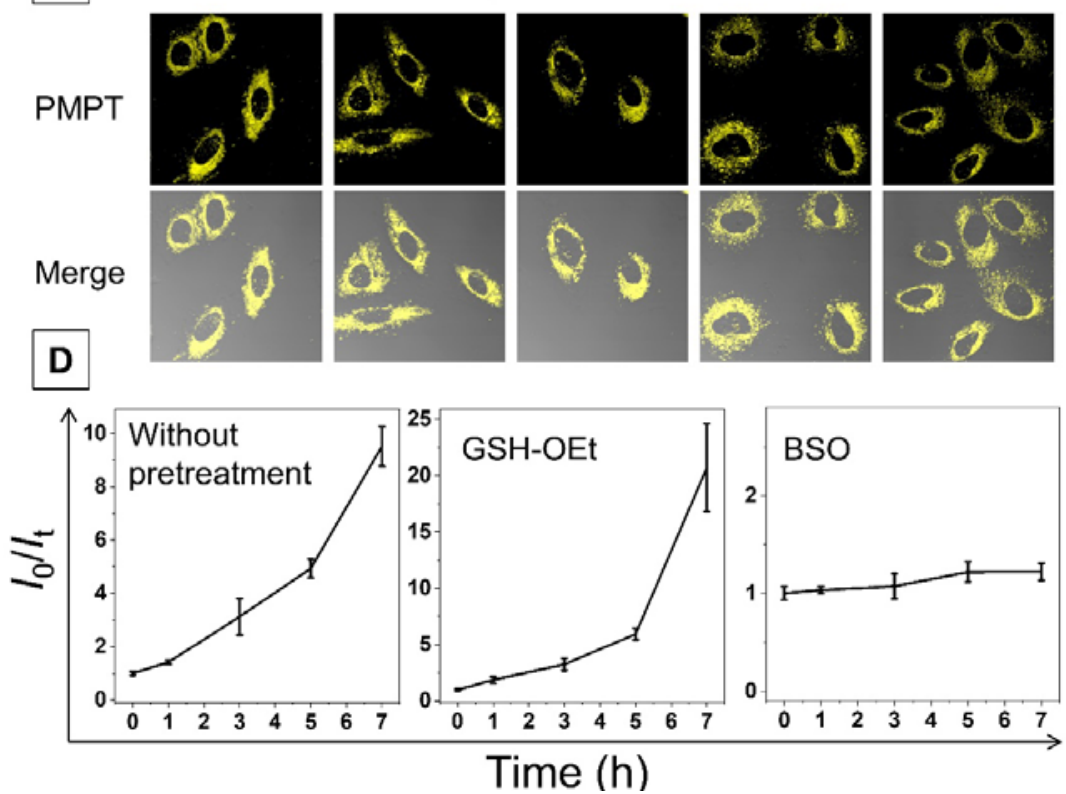

Figure S28. The fluorescence signal of PyTPE was observed by CLSM after PMPT micelles were incubated with HeLa cells for 2 h. (A) HeLa cells without pretreatment, (B) pretreated with glutathione monoester (GSH-OEt) and (C) buthionine sulfoximine (BSO), respectively. (D) Their quantitative analysis of $I_{0} / I_{\mathrm{h}}$ of PyTPE $(\mathrm{n}=3)$ for CLSM images. PyTPE: yellow signal, Ex $=405 \mathrm{~nm}$. Scale bar: $20 \mu \mathrm{m}$. 

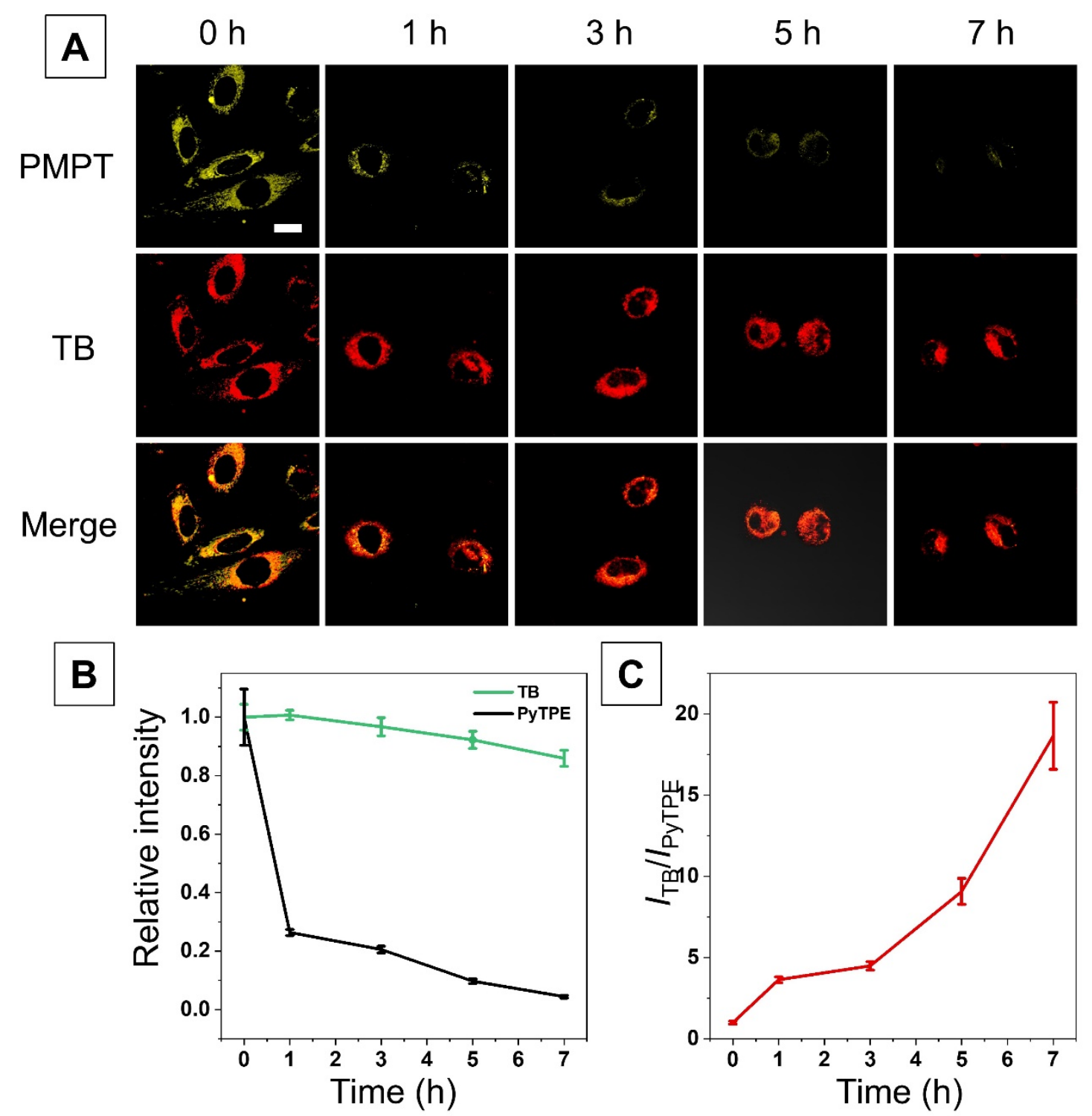

Figure S29. (A) Fluorescence signal of PyTPE and TB was observed by CLSM after TB@PMPT micelles were incubated with HeLa cells for 2 h. HeLa cells were pretreated with GSH-OEt. (B) Quantitative analysis of the fluorescence intensity of PyTPE and TB (n=3). (C) Quantitative analysis of $I_{\mathrm{TB}} / I_{\text {PyтPE }}(\mathrm{n}=3)$ PyTPE: yellow signal, Ex $=405$ nm; TB: red signal, Ex $=543$ nm. Scale bar: $20 \mu \mathrm{m}$. 


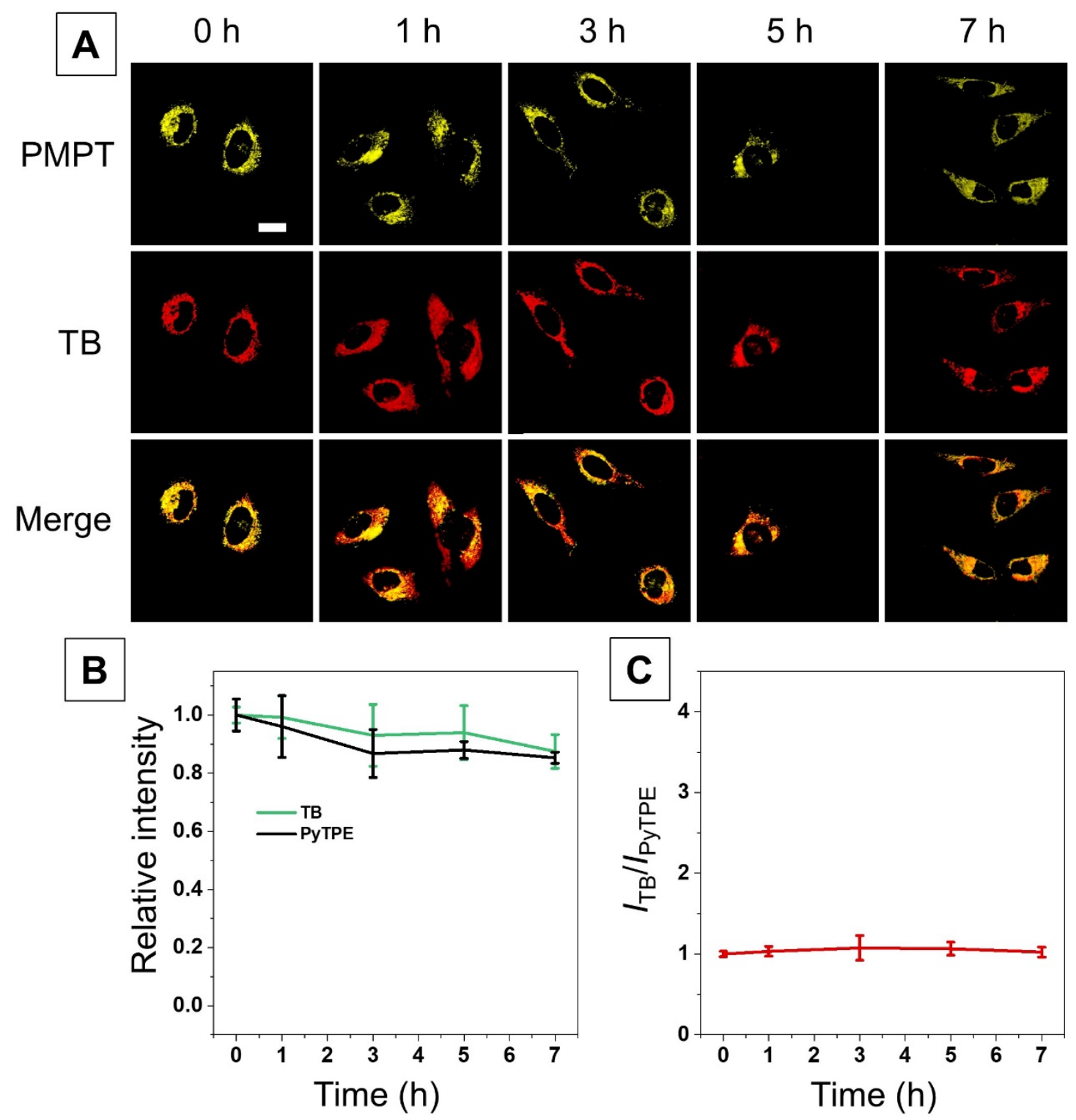

Figure S30. (A) Fluorescence signal of PyTPE and TB was observed by CLSM after

TB@PMPT micelles were incubated with HeLa cells for 2 h. The HeLa cells were pretreated with BSO. (B) Quantitative analysis of the fluorescence intensity of PyTPE and TB (n=3). (C) Quantitative analysis of $I_{\mathrm{TB}} / I_{\mathrm{PyTPE}}(\mathrm{n}=3)$ PyTPE: yellow signal, Ex = 405 nm; TB: red signal, Ex = 543 nm. Scale bar: $20 \mu \mathrm{m}$. 


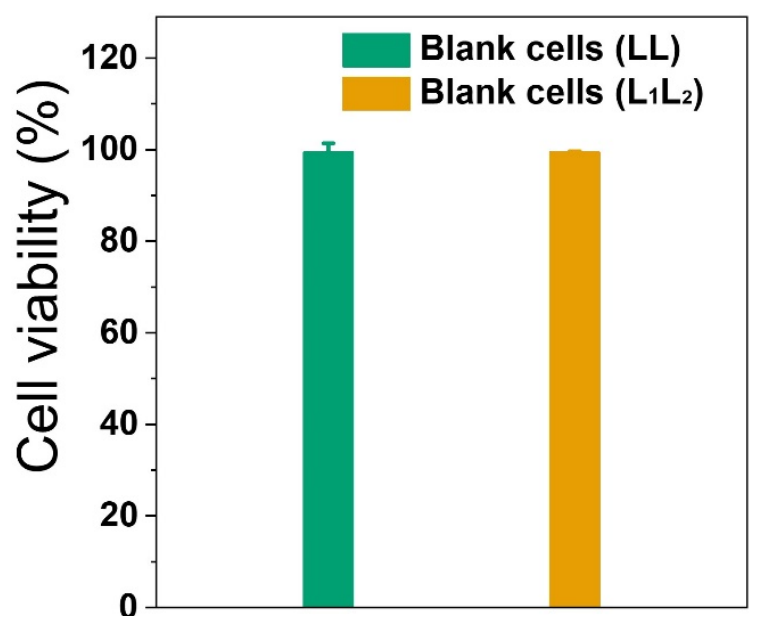

Figure S31. CCK-8 assay of blank HeLa cells (LL, $\left.\mathrm{L}_{1} \mathrm{~L}_{2}\right)$ in HeLa cells after incubation for $48 \mathrm{~h}$. 


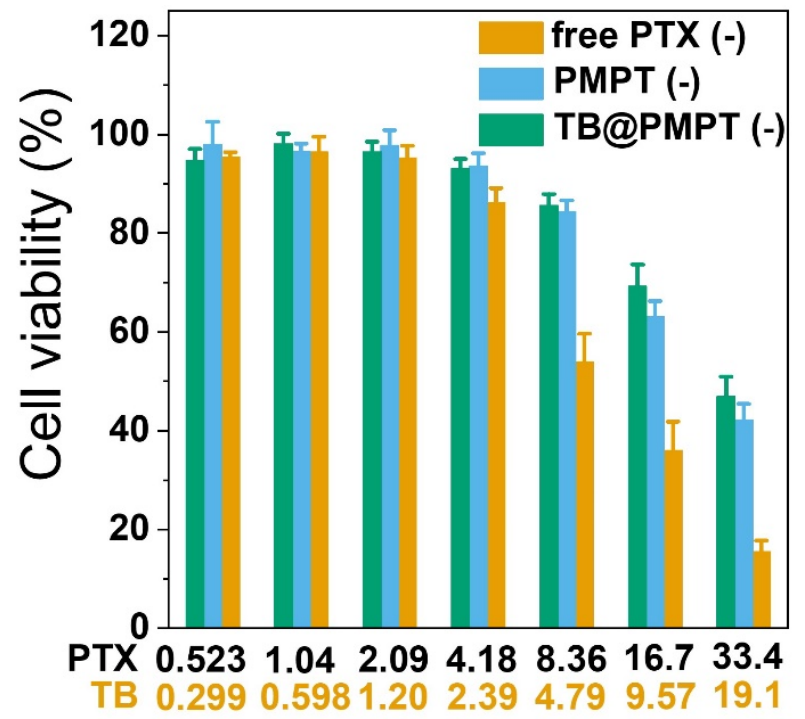

Concentration $\left(\mu \mathrm{g} \mathrm{mL}^{-1}\right)$

Figure S32. CCK-8 assay of free PTX (-), PMPT (-) and TB@PMPT (-) in HeLa cells after incubation for $48 \mathrm{~h}$. 

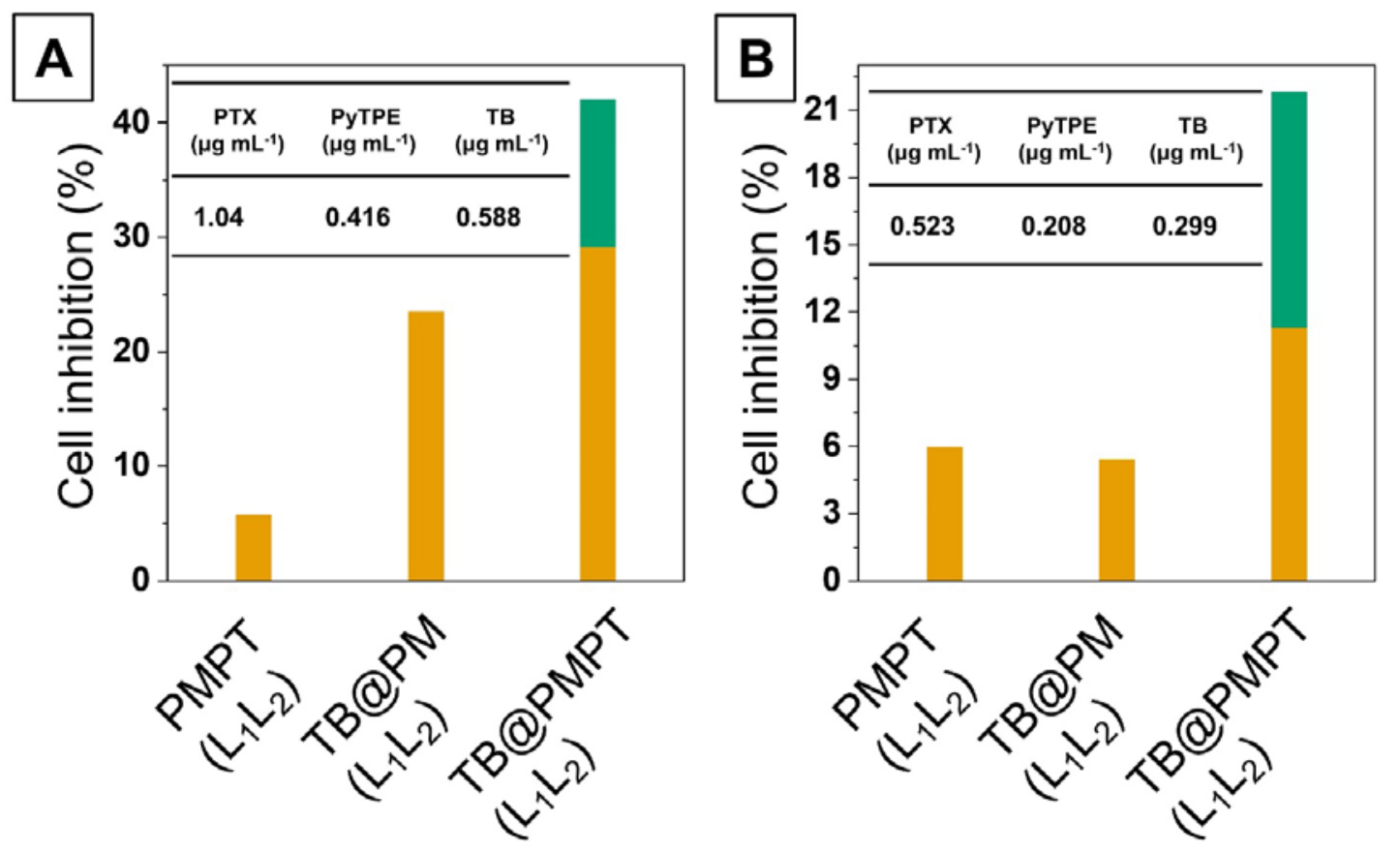

Figure S33. (A, B) The inhibition ratios of TB@PM ( $\left.\mathrm{L}_{1} \mathrm{~L}_{2}\right), \mathrm{PMPT}\left(\mathrm{L}_{1} \mathrm{~L}_{2}\right)$, and TB@PMPT ( $\left.\mathrm{L}_{1} \mathrm{~L}_{2}\right)$ micelles treated cells upon light irradiation (white light, 70mW $\mathrm{cm}^{-2}$ ), respectively. The green bar denotes the additional cell inhibition ratio gained when TB@PMPT $\left(\mathrm{L}_{1} \mathrm{~L}_{2}\right)$ are combined, compared with the sum of TB@PM $\left(\mathrm{L}_{1} \mathrm{~L}_{2}\right)$, $\operatorname{PMPT}\left(\mathrm{L}_{1} \mathrm{~L}_{2}\right)$. 

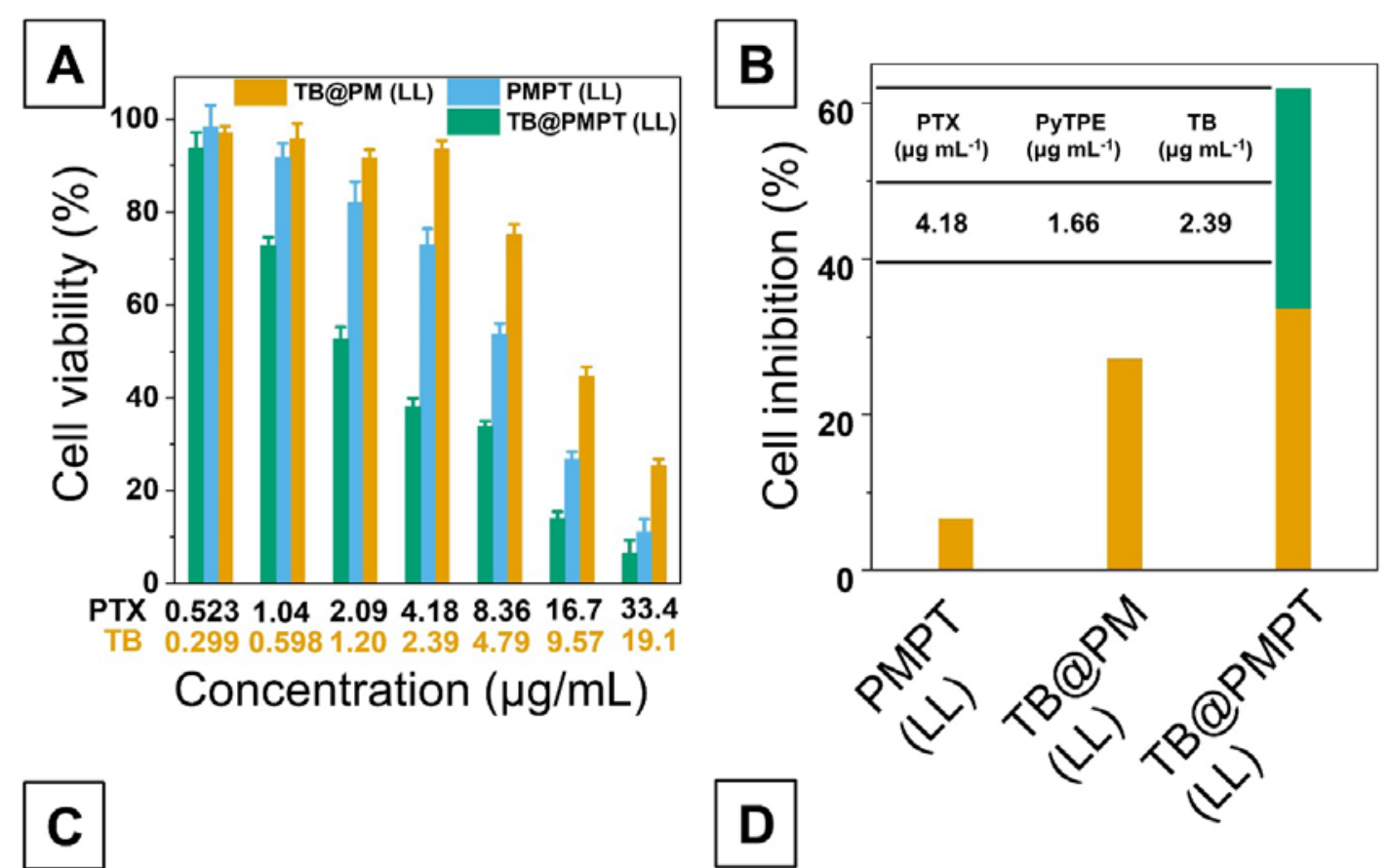

\section{c}
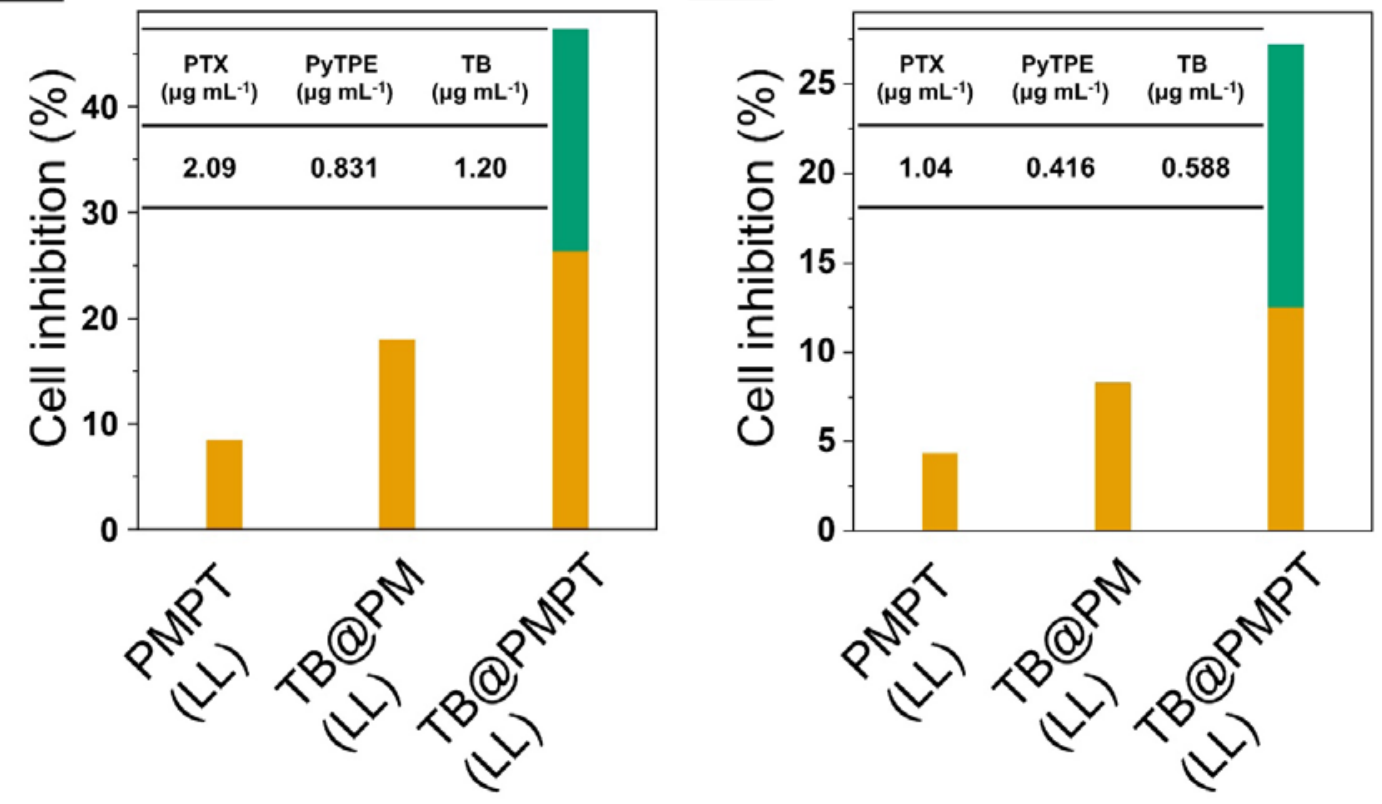

Figure S34. The inhibition ratios of TB@PM (LL), PMPT (LL), and TB@PMPT (LL) micelles treated cells upon light irradiation (white light, $70 \mathrm{~mW} \mathrm{~cm}^{-2}$ ), respectively. The green bar denotes the additional cell inhibition ratio gained when TB@PMPT (LL) are combined, compared with the sum of TB@PM, PMPT (LL). 


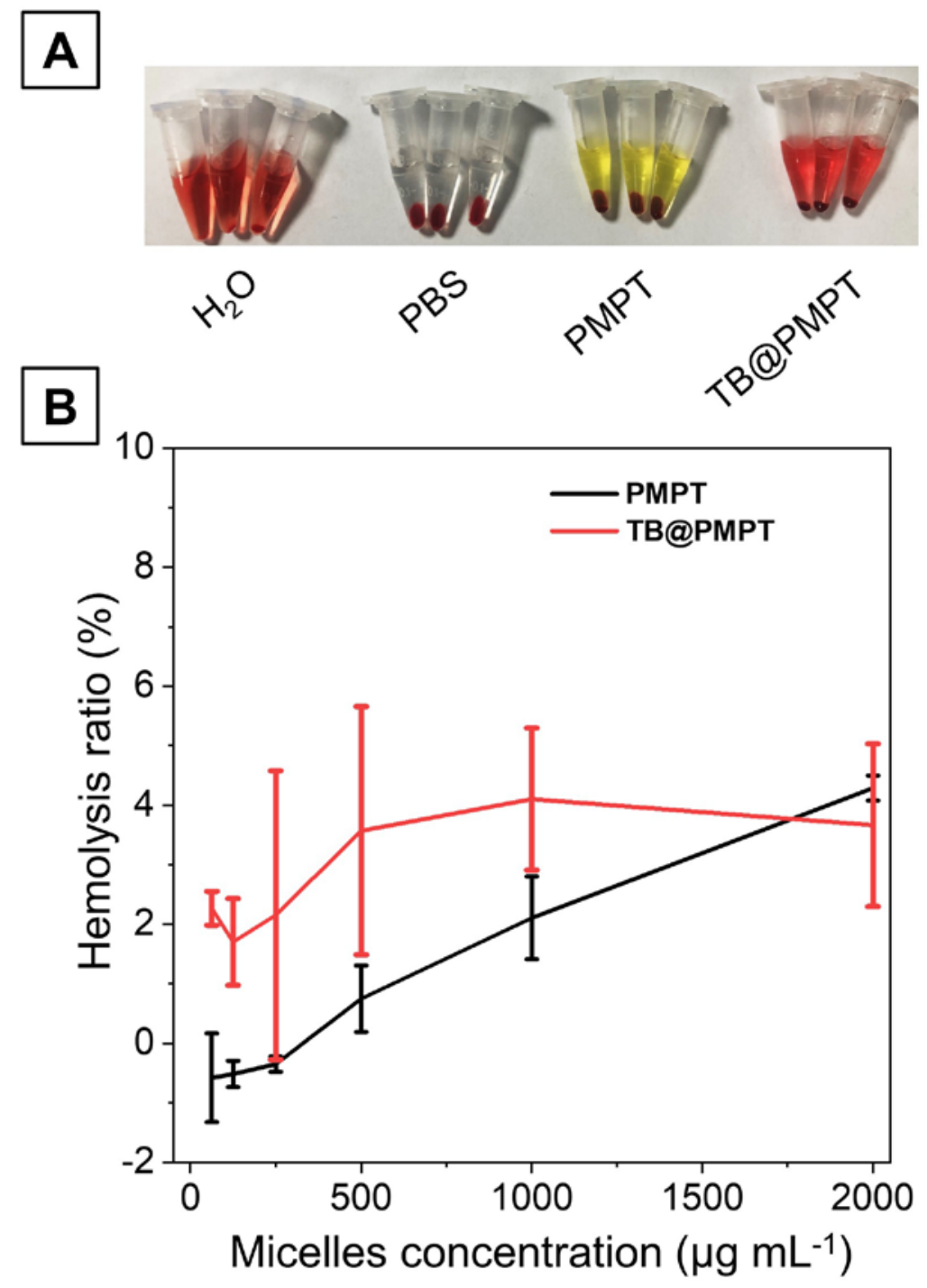

Figure S35. (A) Photographs of hemolysis assay after incubation with ultrapure water (positive control), PBS (negative control), PMPT (2000 mg mL ${ }^{-1}$ ) and TB@PMPT (2000 mg mL ${ }^{-1}$ ) micelles for $6 \mathrm{~h}$ in dark, respectively; (B) The hemolysis ratio induced by PMPT and TB@PMPT micelles with different concentration incubated at $37^{\circ} \mathrm{C}$ for $6 \mathrm{~h}$ in dark. 

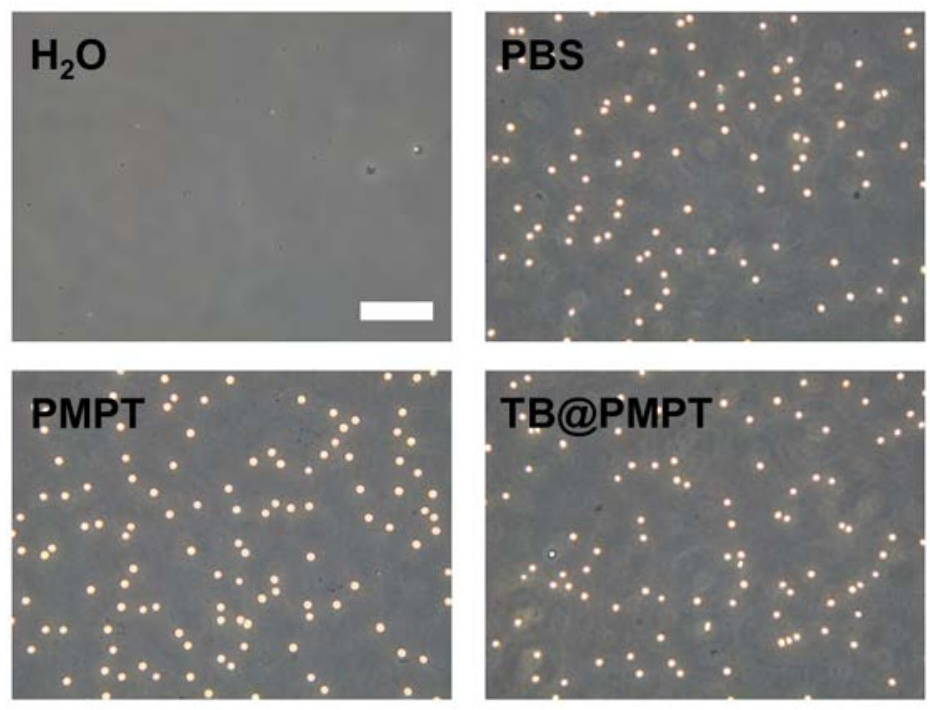

Figure S36. Optical microscopic observation of the dispersion states of the erythrocytes after incubated with $\mathrm{H}_{2} \mathrm{O}$, PBS, PMPT (2000 mg mL $\mathrm{m}^{-1}$ ) and TB@PMPT (2000 mg mL $\left.{ }^{1}\right)$ micelles for $6 \mathrm{~h}$. Scale bar: $50 \mu \mathrm{m}$. 


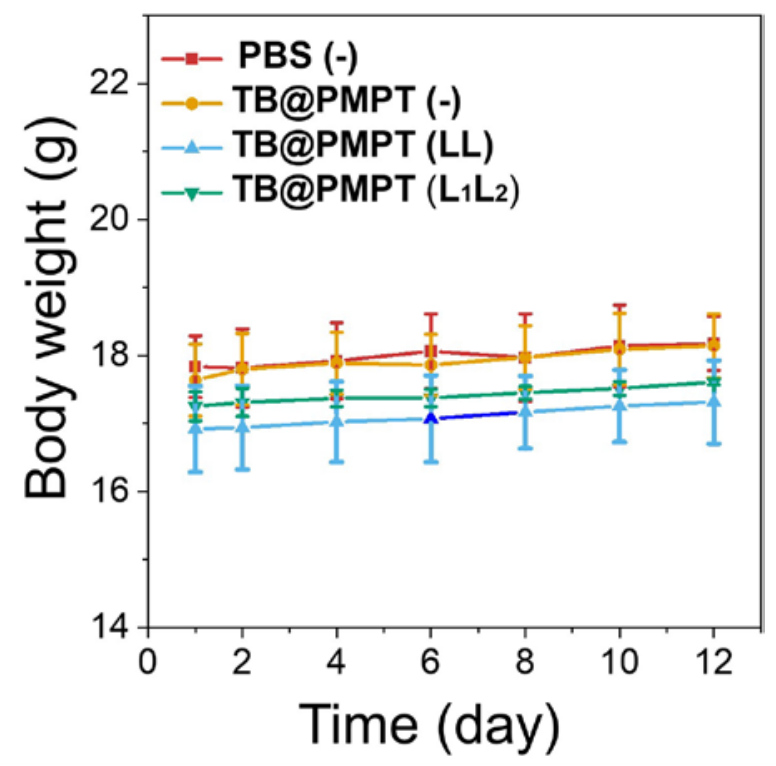

Figure S37. Body weight in the mice after the intravenous of different samples: PBS (-), TB@PMPT (-), TB@PMPT (LL), and TB@PMPT (L1L2). 


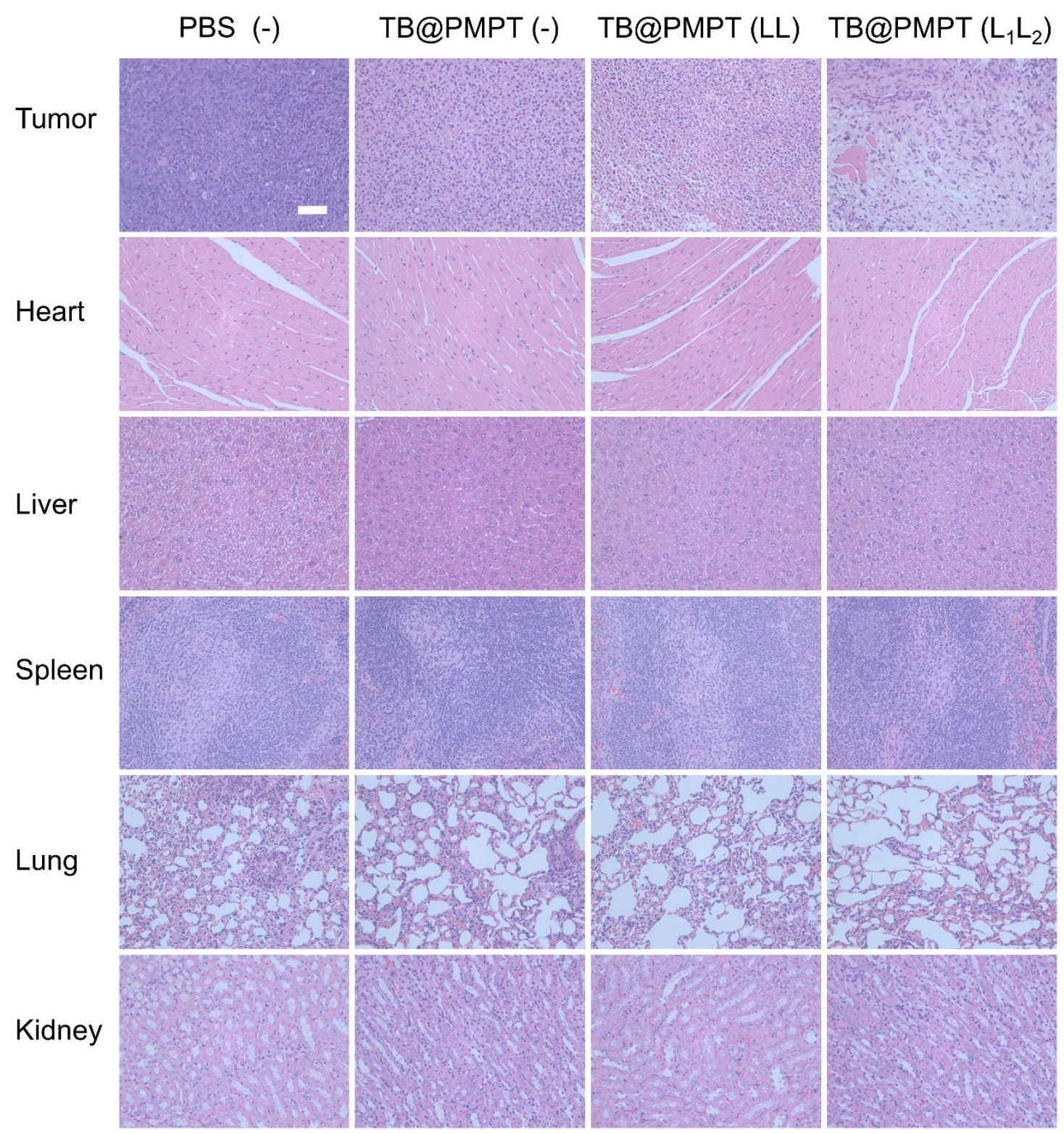

Figure S38. H\&E staining images of the tumor and organs obtained from the mice after different treatments: intravenous injection of different samples: PBS (-), TB@PMPT (-), TB@PMPT (LL) and TB@PMPT (L1L2) micelles. Scale bar: $10 \mu \mathrm{m}$. 